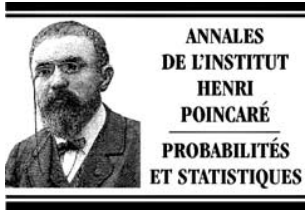

www.elsevier.com/locate/anihpb

\title{
Estimate of spectral gap for continuous gas
}

\author{
Liming $\mathrm{Wu}^{\mathrm{a}, \mathrm{b}}$ \\ ${ }^{a}$ Laboratoire de Math. Appl. CNRS-UMR 6620, Université Blaise Pascal, 63177 Aubière, France \\ ${ }^{\mathrm{b}}$ Department of Math., Wuhan University, 430072 Hubei, China
}

Received 13 February 2003; received in revised form 23 October 2003; accepted 18 November 2003

Available online 25 March 2004

\begin{abstract}
Consider the continuous gas in a bounded domain $\Lambda$ of $\mathbb{R}^{d}$, described by a Gibbsian measure $\mu_{\Lambda}^{\eta}$ associated with a pair interaction $\varphi$, the inverse temperature $\beta$, the activity $z>0$, and the boundary condition $\eta$. When $\varphi$ is nonnegative, we show that the spectral gap of a Glauber type dynamic (i.e., some Markov process reversible with respect to $\left.\mu_{\Lambda}^{\eta}\right)$ in $L^{2}\left(\mu_{\Lambda}^{\eta}\right)$ is bounded from below by $1-z \int_{\mathbb{R}^{d}}\left|1-e^{-\beta \varphi(y)}\right| d y$ and from above by $1+z \int_{\mathbb{R}^{d}}\left|1-e^{-\beta \varphi(y)}\right| d y$, independent of $\Lambda$ and $\eta$. This result improves a previous work by L. Bertini et al. (2002) and is extended also to the hard core case. Our approach consists to approximate the continuous gas model by the discrete spin model and to apply the $M-\varepsilon$ theorem of Ligget. Some other results such as uniqueness, exponential convergence of the Glauber dynamic w.r.t. norms of Ligget's type are also obtained.
\end{abstract}

(c) 2004 Elsevier SAS. All rights reserved.

\section{Résumé}

On considère un gaz continu dans un domaine borné $\Lambda$ de $\mathbb{R}^{d}$, décrit par la mesure de Gibbs $\mu_{\Lambda}^{\eta}$ associée à l'interaction paire $\varphi$, la température inverse $\beta$, l'activité $z>0$ et la condition au bord $\eta$. Quand $\varphi \geqslant 0$, nous démontrons que le trou spectral $\lambda_{1}$ d'une dynamique du type Glauber (i.e., un processus de Markov réversible par rapport à $\left.\mu_{\Lambda}^{\eta}\right)$ dans $L^{2}\left(\mu_{\Lambda}^{\eta}\right)$ vérifie $1-z \int_{\mathbb{R}^{d}}\left|1-e^{-\beta \varphi(y)}\right| d y \leqslant \lambda_{1} \leqslant 1+z \int_{\mathbb{R}^{d}}\left|1-e^{-\beta \varphi(y)}\right| d y$, indépendamment de $\Lambda$ et de $\eta$. Ce résultat améliore le travail de Bertini et al. (2002), et est généralisé au cas de corps durs. Notre méthode consiste à approcher le gaz continu par un modèle de spin discret auquel le théorème $M-\varepsilon$ de Ligget s'applique. Nous établissons également l'unicité de la dynamique de Glauber et sa convergence exponentielle par rapport aux normes du type Ligget.

(c) 2004 Elsevier SAS. All rights reserved.

MSC: 60J75; 60G57; 82B20; 82C20

Keywords: Poincaré inequality; Gibbs measures; Birth and death processes

\section{Introduction}

In statistical mechanics, relations between the mixing properties of the Gibbs measure and the exponential speed at which the associated Glauber dynamics relaxes to equilibrium are a fascinating and important object. For lattice

E-mail address: Li-Ming.Wu@ math.univ-bpclermont.fr (L. Wu). 
spin systems with compact spin space, Stroock and Zegarlinski, in their important and difficult work [18,19] proved the equivalence between the Poincaré inequality, the log-Sobolev inequality, and the Dobrushin-Shlosman mixing condition for the Gibbs measure, both for the Glauber dynamics of pure jumps type or of diffusion type. See $\mathrm{Lu}$ and Yau [12] and Martinelli [9] for further development and the recent work by F. Cesi [5] for a simplified proof. The (partial) extension of their impressive results to unbounded spin case for Glauber dynamics of diffusion type is carried out by Bodineau and Heffler [2,3], Ledoux [10], Yoshida [23] etc.

In this work we are interested in the same question when the discrete lattice $\mathbb{Z}^{d}$ is replaced by the continuum $\mathbb{R}^{d}$ (i.e., gas instead of crystals in physics language). By decomposition $\mathbb{R}^{d}:=\bigcup_{\mathbf{k} \in \mathbb{Z}^{d}} \mathbf{k}[0,1)^{d}$, we may regard this continuous gas model as a lattice model with unbounded spin (and with unbounded interaction). For continuous gas, L. Bertini et al. [4] establish the spectral gap existence of a Glauber dynamic for high temperature or low activity. Let us present this interesting work briefly.

Let $\Lambda$ be a bounded domain in $\mathbb{R}^{d}$. Given the boundary condition $\eta$ outside of $\Lambda$, consider the Gibbs measure $\mu_{\Lambda}^{\eta}$ in $\Lambda$ associated with a "stable" pair interaction $\varphi: \mathbb{R}^{d} \rightarrow(-\infty,+\infty]$, activity $z>0$ and the inverse temperature $\beta$ (see the next section for precise definition). Under the following assumptions

(H1) $\varphi \geqslant 0$ and $\varphi$ is even;

(H2) $\varphi$ is of finite range, i.e., $\varphi(x)=0$ if $|x|>r$ for some finite $r>0$;

(H3) $z \int_{\mathbb{R}^{d}}\left(1-e^{-\beta \varphi(y)}\right) d y<\frac{1}{3 e}$ (i.e., condition (CE) in [4]);

Bertini, Cancrini and Cesi [4] (Theorem 2.2) establish that $\mu_{\Lambda}^{\eta}$ for all rectangles $\Lambda$ satisfies the Poincaré inequality

$$
G \mu_{\Lambda}^{\eta}(f, f) \leqslant \mathcal{E}_{\Lambda}^{\eta}(f, f), \quad \forall f
$$

where the constant $G=G(z, \beta, r)>0$ is independent of $\eta$ and $\Lambda$, and $\mathcal{E}_{\Lambda}^{\eta}$ is a quite natural Dirichlet form on $L^{2}\left(\mu_{\Lambda}^{\eta}\right)$ generating the Glauber dynamic (which is a birth-death Markov process reversible w.r.t. $\mu_{\Lambda}^{\eta}$, see Sections 2,3). Their main idea is:

1) a quasi-factorization of the variance;

2) to establish an exponential decay of correlation between $f$ and $g$ when their "supports" are sufficiently separated by condition (H3) and cluster expansion;

3) the iterative method by doubling the volume and a delicate geometric consideration.

Their result so obtained does not, seems - it however, yield a robust estimate of the spectral gap constant $\lambda_{1}$, like most known results in $[18,19,23,12]$ issued of the iterative method (we emphasize that some explicit spectral gap estimates are given by Bodineau and Heffler [2,3] and Ledoux [10]). The reader can compare their (H3) with the following classical estimate of the convergence radius $R$ of the cluster expansion of the pression $p=p(z)$ (thermodynamic limit) in terms of the activity $z$ ([17], Theorem 4.5.3):

$$
\frac{1}{e} \leqslant R \int_{\mathbb{R}^{d}}\left(1-e^{-\beta \varphi(y)}\right) d y \leqslant 1 .
$$

So their result can be roughly read as the spectral gap existence when $|z|<R / 3$.

The main aim of this paper is to improve their result (1.1). Indeed our main result (see Theorem 2.1) says that for nonnegative $\varphi$, the best constant $G$ for (1.1), denoted by $\lambda_{1}$ (i.e., the spectral gap), satisfies

$$
1-z \int_{\mathbb{R}^{d}}\left(1-e^{-\beta \varphi(y)}\right) d y \leqslant \lambda_{1} \leqslant 1+z \int_{\mathbb{R}^{d}}\left(1-e^{-\beta \varphi(y)}\right) d y
$$

without the finite range condition (H2). Hence $\lambda_{1}$ is uniformly lower bounded once if $z \int_{\mathbb{R}^{d}}\left(1-e^{-\beta \varphi(y)}\right) d y<1$, a condition weaker than (H3), and sharp in the viewpoint of (1.2). Moreover we extend this result to the hard core case. 
The estimate above yields $\lambda_{1}=1$ when $\varphi=0$ (i.e., the free case), a well known result (to all specialists on Malliavin Calculus over the Poisson space). See Ané and Ledoux [1] and the author [21] for modified log-Sobolev inequalities which are stronger than the Poincaré inequality.

Our method will be completely different, and more classical in some sense. Indeed our idea is inspired by the classical Poisson limit theorem, i.e., a Poisson distribution is the weak limit of laws of sums of i.i.d. Bernoulli random variables. Then it is not surprise that we can approximate the Glauber dynamic associated with $\mu_{\Lambda}^{\eta}$ by the spin models on $\{0,1\}^{V}$ for which the Ligget's theorem gives us an explicit exponential rate for the "triple" norm. Hence the key consists to bound the Ligget's constant for the spin models on $\{0,1\}^{V}$ by means of $\varphi$, and to transform that convergence rate in $L^{2}\left(\mu_{\Lambda}^{\eta}\right)$, and fortunately this is possible.

This paper is organized as follows. The next section is devoted to describe the Gibbs measure, the Glauber dynamic and the main result. In Section 3 we solve some uniqueness problems (which are crucial for approximation) and construct the corresponding Markov process. Section 4 is devoted to the approximation of continuous gas by discrete spin models, which is the crucial part of this paper. As consequence, the spectral gap result is derived in Section 5, together with the exponential convergence in other senses (than $L^{2}$ ).

\section{Main result}

\subsection{Gibbs measure}

Let $\Omega$ be the space of all point measures $\omega=\sum_{i} \delta_{x_{i}}$ (finite or countable) with $x_{i}$ different in $\mathbb{R}^{d}$, which are moreover Radon measures (i.e., finite particles in compact subsets), where $\delta_{x}$ denotes the Dirac measure at $x$. Let $\mathcal{F}_{A}:=\sigma(\omega(B) ; B(B$ Borelian $) \subset A)$ for each $A \in \mathcal{B}\left(\mathbb{R}^{d}\right)$, the Borel $\sigma$-filed of $\mathbb{R}^{d}$ and $\mathcal{F}=\mathcal{F}_{\mathbb{R}^{d}}$. Given the activity $z>0$, let $P$ be the law of the Poisson point process on $\mathbb{R}^{d}$ with intensity measure $z d x$, i.e., a probability measure on $(\Omega, \mathcal{F})$ characterized by

(i) $P(\omega(A)=k)=e^{-z|A|} \frac{(z|A|)^{k}}{k !}, \forall k \in \mathbb{N}$ for any $A \in \mathcal{B}\left(\mathbb{R}^{d}\right)$; here and throughout this paper $|A|$ designs the Lebesgue volume of $A$;

(ii) If $A_{i} \in \mathcal{B}\left(\mathbb{R}^{d}\right), i=1, \ldots, n$, are disjoint, then $\omega\left(A_{i}\right), i=1, \ldots, n$, are $P$-independent.

Throughout this paper the pair interaction $\varphi: \mathbb{R}^{d} \rightarrow(-\infty,+\infty]$ will be a Borel-measurable even function which is stable ([17]), i.e., $\exists B \geqslant 0$ such that

$$
H(\omega):=\sum_{1 \leqslant i<j \leqslant n} \varphi\left(x_{i}-x_{j}\right) \geqslant-B n, \quad \forall \omega=\sum_{j=1}^{n} \delta_{x_{j}}, n \geqslant 1 \text { (stability). }
$$

We assume often also that $\varphi$ is regular [17], i.e.,

$$
\int_{\mathbb{R}^{d}}\left|1-e^{-\beta \varphi(y)}\right| d y<+\infty
$$

where $\beta=(\kappa T)^{-1}>0$ is the inverse temperature. Recall that (see [17]) the stability condition is a necessary and sufficient condition for defining the (free boundary) Gibbs measures on bounded domains $\Lambda$. Moreover for a stable pair interaction $\varphi, \varphi(x)=H\left(\delta_{x}+\delta_{0}\right) \geqslant-2 B$ by (2.1), and then the regularity condition (2.2) is equivalent to the integrability of $\varphi$ outside of some finite measure set (e.g. $[\varphi \geqslant 1])$.

Given a bounded open and non-empty domain $\Lambda \subset \mathbb{R}^{d}$ and $\omega \in \Omega$, let $\omega_{\Lambda}=\sum_{x_{i} \in \Lambda \bigcap \operatorname{supp}(\omega)} \delta_{x_{i}}$ be the restriction of the measure $\omega$ to $\Lambda$, and $\Omega_{\Lambda}=\left\{\omega_{\Lambda} ; \omega \in \Omega\right\}$. The image measure $P_{\Lambda}$ of $P$ by $\omega \rightarrow \omega_{\Lambda}$ is the law of Poisson point process on $\Lambda$ with intensity measure $z d x$. 
We say that an element $\eta$ of $\Omega$ is in $\Omega^{\varphi}$, if

$$
x \rightarrow \int_{\mathbb{R}^{d}} \varphi^{-}(x-y) d \eta(y) \text { is locally bounded on } \mathbb{R}^{d} .
$$

When $\varphi \geqslant 0$ or is of finite range, we have of course $\Omega^{\varphi}=\Omega$.

The Gibbs measure in $\Lambda$ for a given boundary condition $\eta \in \Omega^{\varphi}$ on $\Lambda^{c}$ is a probability measure on $\left(\Omega_{\Lambda}, \mathcal{F}_{\Lambda}\right)$ given by

$$
\mu_{\Lambda}^{\eta}\left(d \omega_{\Lambda}\right):=\frac{1}{Z(\Lambda, \eta)} e^{-\beta H_{\Lambda}^{\eta}\left(\omega_{\Lambda}\right)} P_{\Lambda}\left(d \omega_{\Lambda}\right)
$$

where

$$
H_{\Lambda}^{\eta}\left(\omega_{\Lambda}\right):=H\left(\omega_{\Lambda}\right)+\int_{\Lambda} \omega(d x) \int_{\Lambda^{c}} \varphi(x-y) \eta(d y)
$$

is the Hamiltonian $\left(H\left(\omega_{\Lambda}\right)\right.$ being given in $\left.(2.1)\right)$, and

$$
Z(\Lambda, \eta):=\int_{\Omega_{\Lambda}} e^{-\beta H_{\Lambda}^{\eta}\left(\omega_{\Lambda}\right)} d P_{\Lambda}\left(\omega_{\Lambda}\right)
$$

is the normalization constant. Remark that $H_{\Lambda}^{\eta}(\mathbf{0})=0$ where $\mathbf{0}$ denotes the zero measure (or the vide state) and by the stability condition,

$$
H_{\Lambda}^{\eta}\left(\omega_{\Lambda}\right) \geqslant-B N_{\Lambda}(\omega)+\int_{\Lambda} h^{\eta}(x) d \omega(x)
$$

where $N_{\Lambda}(\omega)=\omega(\Lambda)$ is the number of particles in the configuration $\omega_{\Lambda}$, and

$$
h^{\eta}(x)=\int_{\Lambda^{c}} \varphi(x-y) \eta_{\Lambda^{c}}(d y)
$$

which is bounded from below on $\Lambda$ by (2.3). Hence $Z(\Lambda, \eta)$ is a finite positive constant, $\mu_{\Lambda}^{\eta}$ is then a well defined probability on $\Omega_{\Lambda}$, and moreover

$$
\frac{d \mu_{\Lambda}^{\eta}}{d P_{\Lambda}} \in \bigcap_{1 \leqslant p<+\infty} L^{p}\left(P_{\Lambda}\right)
$$

\subsection{Generator of the Glauber dynamic}

Let $r \mathcal{F}$ be the space of real $\mathcal{F}$-measurable functions, and $b \mathcal{F}$ the space of those $F \in r \mathcal{F}$ which are moreover bounded. For any $r \in r \mathcal{F}$, according to Picard [16] consider the difference operators

$$
\begin{aligned}
& D_{x}^{+} F(\omega):=F\left(\varepsilon_{x}^{+} \omega\right)-F(\omega), \quad \varepsilon_{x}^{+} \omega:=\omega+1_{x \notin \operatorname{supp} \omega} \delta_{x} ; \\
& D_{x}^{-} F(\omega):=F\left(\varepsilon_{x}^{-} \omega\right)-F(\omega), \quad \varepsilon_{x}^{-} \omega:=\omega-1_{x \in \operatorname{supp} \omega} \delta_{x} ; \\
& D_{x} F(\omega):=F\left(\varepsilon_{x}^{+} \omega\right)-F\left(\varepsilon_{x}^{-} \omega\right) .
\end{aligned}
$$

Those resulting functions are measurable jointly on $\mathbb{R}^{d} \times \Omega$. Recall that $D_{x}^{+}$(or $D_{x}$ ) plays the same role in the Malliavin calculus over the Poisson space as the Malliavin derivative on the Wiener space $([16,21]$ and references therein).

We shall study the Glauber dynamic employed in [4], which is formally generated by the pre-generator 


$$
\begin{aligned}
\mathcal{L}_{\Lambda}^{\eta} F\left(\omega_{\Lambda}\right) & =\int_{\Lambda} D_{x}^{-} F\left(\omega_{\Lambda}\right) \omega_{\Lambda}(d x)+z \int_{\Lambda} e^{-\beta D_{x}^{+} H_{\Lambda}^{\eta}\left(\omega_{\Lambda}\right)} D_{x}^{+} F\left(\omega_{\Lambda}\right) d x \\
& =-\int_{\Lambda} e^{-\beta D_{x}^{+} H_{\Lambda}^{\eta}\left(\omega_{\Lambda}\right)} D_{x} F\left(\omega_{\Lambda}\right)\left(\omega_{\Lambda}(d x)-z d x\right), \quad \forall F \in b \mathcal{F}_{\Lambda}
\end{aligned}
$$

(recalling $\left.D_{x}^{+} H_{\Lambda}^{\eta}\left(\omega_{\Lambda}\right)=0, \forall x \in \operatorname{supp} \omega_{\Lambda}\right)$. Its dynamic can be intuitively described as follows: if the configuration of the system is $\omega_{\Lambda}$ at time $\mathrm{t}$, each particle in $\omega_{\Lambda}$ will be killed at rate $d t$, and a new particle will be born at $x \in \Lambda$ with rate $z e^{-\beta D_{x}^{+} H_{\Lambda}^{\eta}\left(\omega_{\Lambda}\right)} d x d t$.

Let $\|F\|_{u}=\sup _{\omega \in \Omega_{\Lambda}}|F(\omega)|$. Since $\left|\int_{\Lambda} D_{x}^{-} F\left(\omega_{\Lambda}\right) \omega_{\Lambda}(d x)\right| \leqslant 2\|F\|_{u} \cdot N_{\Lambda}(\omega)$ and

$$
D_{x}^{+} H_{\Lambda}^{\eta}\left(\omega_{\Lambda}\right)=\int_{\Lambda} \varphi(y-x)\left(\omega_{\Lambda}(d y)+\eta_{\Lambda^{c}}(d y)\right) \geqslant-2 B N_{\Lambda}(\omega)+h^{\eta}(x), \quad \forall x \notin \operatorname{supp} \omega_{\Lambda}
$$

where $h^{\eta}(x)$ given in (2.5) is lower bounded, then it is easy to verify that $\mathcal{L}_{\Lambda}^{\eta} F \in r \mathcal{F}_{\Lambda}$ and $\mathcal{L}_{\Lambda}^{\eta} F \in$ $\bigcap_{1 \leqslant p<+\infty} L^{p}\left(\mu_{\Lambda}^{\eta}\right)$ for all $F \in b \mathcal{F}_{\Lambda}$. Moreover if $F_{1}=F_{2}, \mu_{\Lambda}^{\eta}$-a.s., $\mathcal{L}_{\Lambda}^{\eta} F_{1}=\mathcal{L}_{\Lambda}^{\eta} F_{2}, \mu_{\Lambda}^{\eta}$-a.s. (by [16], Section 4). In particular $\mathcal{L}$ is a well defined operator on $L^{p}\left(\mu_{\Lambda}^{\eta}\right)$ with domain $b \mathcal{F}_{\Lambda}$ (in which each element represent a class of $\mu_{\Lambda}^{\eta}$-equivalent functions, by usual convention). In further by Picard [16] (Proposition 6 and Théorème 2), for all $F, G \in b \mathcal{F}_{\Lambda}$, we have by a simple calculus

$$
\begin{aligned}
\left\langle F,-\mathcal{L}_{\Lambda}^{\eta} G\right\rangle_{\mu_{\Lambda}^{\eta}} & =\int_{\Omega_{\Lambda}} d \mu_{\Lambda}^{\eta}\left(\omega_{\Lambda}\right) \sum_{x \in \operatorname{supp} \omega} D_{x}^{-} F\left(\omega_{\Lambda}\right) D_{x}^{-} G\left(\omega_{\Lambda}\right) \\
& =\int_{\Omega_{\Lambda}} d \mu_{\Lambda}^{\eta}\left(\omega_{\Lambda}\right) \int_{\Lambda} e^{-\beta D_{x}^{+} H_{\Lambda}^{\eta}\left(\omega_{\Lambda}\right)} D_{x}^{+} F\left(\omega_{\Lambda}\right) D_{x}^{+} G\left(\omega_{\Lambda}\right) z d x \\
& =: \mathcal{E}_{\Lambda}^{\eta}(F, G) .
\end{aligned}
$$

(L. Bertini et al. [4] (Proposition 2.1) has indicated this fact when $\varphi \geqslant 0$.) Then $\left(-\mathcal{L}_{\Lambda}^{\eta}, b \mathcal{F}_{\Lambda}\right)$ is nonnegative definite, symmetric operator on $L^{2}\left(\mu_{\Lambda}^{\eta}\right)$. Hence $\mathcal{E}_{\Lambda}^{\eta}$ is a closable form and its closure $\left(\mathcal{E}_{\Lambda}^{\eta}, \mathbb{D}\left(\mathcal{E}_{\Lambda}^{\eta}\right)\right)$ is a Dirichlet form on $L^{2}\left(\mu_{\Lambda}^{\eta}\right)$, generating a symmetric Markov semigroup $\left(P_{t}^{\Lambda, \eta}\right)$ on $L^{2}\left(\mu_{\Lambda}^{\eta}\right)$ such that $P_{t}^{\Lambda, \eta} 1=1$, $\mu_{\Lambda^{-}}^{\eta}$ a.s. (since $\mathcal{L}_{\Lambda}^{\eta} 1=0$ ). The last symmetric Markov semigroup, whose generator is the Friedrichs extension of $\left(\mathcal{L}_{\Lambda}^{\eta}, b \mathcal{F}_{\Lambda}\right)$, is the Glauber dynamic used in [4] and in this work. Of course $\left(P_{t}^{\Lambda, \eta}\right)$ is also a strongly continuous semigroup of contractions on $L^{p}\left(\mu_{\Lambda}^{\eta}\right)$, whose generator will be denoted by $\left(\mathcal{L}_{\Lambda}^{\eta}, \mathbb{D}_{p}\left(\mathcal{L}_{\Lambda}^{\eta}\right)\right)\left(\mathbb{D}_{p}\left(\mathcal{L}_{\Lambda}^{\eta}\right)\right.$ being its domain in $\left.L^{p}\left(\mu_{\Lambda}^{\eta}\right)\right)$.

Notice that the same kind of model is studied by Olla and Tremoulet [15] (2003).

\subsection{Main result}

Theorem 2.1. Let $\varphi: \mathbb{R}^{d} \rightarrow(-\infty,+\infty]$ be an even measurable function on $\mathbb{R}^{d}$, which is both stable (2.1) and regular (2.2). Assume either

(C1) $\varphi \geqslant 0$ (nonnegative potential); or

(C2) for some $r_{h c}>0, \varphi(x)=+\infty$ if $|x|<r_{h c}$ (hard core) $\left(|x|\right.$ denotes the Euclidean norm of $\left.x \in \mathbb{R}^{d}\right)$ and for some $B \geqslant 0$,

$$
\sum_{i=1}^{n} \varphi\left(x_{i}\right) \geqslant-2 B
$$

for all $n \geqslant 1$ and all $\left(x_{1}, \ldots, x_{n}\right) \in\left(\mathbb{R}^{d}\right)^{n}$ such that $\left|x_{i}-x_{j}\right| \geqslant r_{h c}$ for $i \neq j$. 
Then for any bounded (non-empty) open domain $\Lambda$ of $\mathbb{R}^{d}$ and for any $\eta \in \Omega^{\varphi}$, the spectral gap $\lambda_{1}$ of $\left(-\mathcal{L}_{\Lambda}^{\eta}, \mathbb{D}_{2}\left(\mathcal{L}_{\Lambda}^{\eta}\right)\right)$ in $L^{2}\left(\mu_{\Lambda}^{\eta}\right)$, i.e. the best constant $\lambda_{1} \geqslant 0$ such that

$$
\lambda_{1} \mu_{\Lambda}^{\eta}(F, F) \leqslant \mathcal{E}_{\Lambda}^{\eta}(F, F), \quad \forall F \in \mathbb{D}\left(\mathcal{E}_{\Lambda}^{\eta}\right)
$$

satisfies

$$
1-z e^{2 \beta B} \int_{\mathbb{R}^{d}}\left|1-e^{-\beta \varphi(x)}\right| d x \leqslant \lambda_{1} \leqslant 1+z e^{\beta B} \int_{\mathbb{R}^{d}}\left(1-e^{-\beta \varphi^{+}(x)}\right) d x
$$

where the constant $B$ is given by condition (2.11) (of course $B=0$ if $\varphi \geqslant 0$ ), $\mu_{\Lambda}^{\eta}(F, G)$ denotes the covariance of $F, G$ under $\mu_{\Lambda}^{\eta}$, and $\left(\mathcal{E}_{\Lambda}^{\eta}, \mathbb{D}\left(\mathcal{E}_{\Lambda}^{\eta}\right)\right.$ is the closure of the form $\left(\mathcal{E}_{\Lambda}^{\eta}, b \mathcal{F}_{\Lambda}\right)$ given in (2.10).

Remarks 2.2. Under the hard core assumption in (C2), condition (2.11) is verified once if there is a nonnegative decreasing function $h:\left[r_{h c},+\infty\right) \rightarrow \mathbb{R}^{+}$such that

$$
\int_{r_{h c}}^{\infty} h(r) r^{d-1} d r<+\infty \quad \text { and } \quad \varphi(x) \geqslant-h(|x|), \quad \forall x \in \mathbb{R}^{d},|x| \geqslant r_{h c}
$$

(see [17], Section 3.2.5, pp. 37-38). Moreover (C2) implies the stability condition (2.1) with the same constant $B$.

Remarks 2.3. Let $p(z):=\beta^{-1} \lim _{\Lambda \uparrow \mathbb{R}^{d}} \frac{1}{\Lambda \Lambda} \log Z(\Lambda, \mathbf{0})$ (thermodynamic limit in the sense of Von Hove, see [17]) be the pressure function. In the nonnegative case $(\mathrm{C} 1)$, our condition about the existence of spectral gap is sharp in the point of view of the cluster expansion estimate (1.2) (but perhaps not all sharp in reality, because $p(z)$ may be analytical for real $z \geqslant R)$. And the spectral gap result above suggests that $R=R_{0}:=\left(\int_{\mathbb{R}^{d}}\left(1-e^{-\beta \varphi(y)}\right) d y\right)^{-1}$ or at least $p(z)$ is analytical for $z \in\left[0, R_{0}\right.$ ), a claim (perhaps known) that I do not know how to prove it.

In the general stable and regular case, the classical estimate of the convergence radius $R$ of the pressure functional $p(z)$ in terms of the activity $z$ verifies

$$
R \cdot e^{2 \beta B} \int_{\mathbb{R}^{d}}\left|1-e^{-\beta \varphi(x)}\right| d x \geqslant \frac{1}{e}
$$

(see [17], Theorem 4.2.3). And our result suggests that the estimate above may hold with $1 / e$ substituted by 1 on the right hand side above, in the hard core case (C2) (to which we equally have no answer).

An interesting (open) question is to extend Theorem 2.1 to general stable and regular interaction $\varphi$. Our proof seems working only under $(\mathrm{C} 1)$ or $(\mathrm{C} 2)$.

Remarks 2.4. L. Bertini et al. [4] derive, from the spectral gap existence, the exponential decay of correlation $\mu_{\Lambda}^{\eta}(F, G)$ where $F \in b \mathcal{F}_{A}$ and $G \in b \mathcal{F}_{B}$, when the distance between their "supports" $A, B$ is large, illustrating the impetus of spectral gap in the understanding of the mixing properties of the underlying Gibbs measure.

Though our theorem above improves their main result [4] Theorem 2.2, but one main contribution of [4] resides in their approach: the exponential decay of correlation in the form of their Corollary 2.5 implies the spectral gap existence, via their quasi-factorization of variance. Indeed F. Cesi [5] utilizes this approach to give a simplified proof of the Stroock-Zegarlinski's log-Sobolev inequality of the Gibbs measure, but our approach here is valid only for the Poincaré inequality.

Remarks 2.5. The continuous gas model has an essential difference from the lattice spin model with compact spin space: the equivalence between the (uniform) Poincaré inequality and the (uniform) log-Sobolev inequality is lost. In fact it is known since the work of Surgailis [20] on 1984 that even the free Poisson measure $P_{\Lambda}$ does not 
satisfy the log-Sobolev inequality. And Ané and Ledoux [1] and the author [21] prove the modified log-Sobolev inequalities, one of which is the following $L^{1}-\log -$ Sobolev inequality [21]

$$
\operatorname{Ent}_{P_{\Lambda}}(F):=\mathbb{E}^{P_{\Lambda}} F \log \frac{F}{\mathbb{E}^{P_{\Lambda}}(F)} \leqslant \mathbb{E}^{P_{\Lambda}} \int_{\Lambda} D_{x}^{+} F D_{x}^{+} \log F z d x, \quad \forall 0<F \in b \mathcal{F}_{\Lambda} .
$$

This last inequality is equivalent to the exponential decay in the sense of entropy: $\operatorname{Ent}_{P_{\Lambda}}\left(P_{t}^{0} F\right) \leqslant e^{-t} \operatorname{Ent}_{P_{\Lambda}}(F)$, where $P_{t}^{0}=P_{t}^{\Lambda, \eta}$ with $\varphi=0$. And it is equivalent to the usual log-Sobolev inequality when the employed Dirichlet form is of diffusion type (but this interesting equivalence fails for jumps processes: the case here). One can then reasonably hope the equivalence of the Poincare inequality with the $L^{1}$-log-Sobolev inequality above for the continuous gas model here.

Notice that Dai Pra, Paganoni and Posta [6] (2002) establish the $L^{1}-\log -$ Sobolev inequality for the lattice gas with unbounded spin.

\subsection{A guideline to our proof of Theorem 2.1}

For the reader's convenience, let us outline our approach for proving the lower bound of $\lambda_{1}$ in Theorem 2.1. At first notice that

$$
\begin{aligned}
\frac{d}{d t} \mu_{\Lambda}^{\eta}\left(P_{t}^{\Lambda, \eta} F, P_{t}^{\Lambda, \eta} F\right) & =-2 \mathcal{E}_{\Lambda}^{\eta}\left(P_{t}^{\Lambda, \eta} F, P_{t}^{\Lambda, \eta} F\right) \\
& =-2 \int_{\Omega_{\Lambda}} d \mu_{\Lambda}^{\eta}\left(\omega_{\Lambda}\right) \int_{\Lambda} e^{-\beta D_{x}^{+} H_{\Lambda}^{\eta}\left(\omega_{\Lambda}\right)}\left(D_{x}^{+} P_{t}^{\Lambda, \eta} F\left(\omega_{\Lambda}\right)\right)^{2} z d x .
\end{aligned}
$$

If one can prove that for some $c>0$, for all $F$ belonging a $L^{2}\left(\mu_{\Lambda}^{\eta}\right)$-dense class of test functions $\mathcal{D}$,

$$
\left\|D_{x}^{+} P_{t}^{\Lambda, \eta} F\left(\omega_{\Lambda}\right)\right\| \leqslant e^{-c t}\left\|D_{x}^{+} F\left(\omega_{\Lambda}\right)\right\|
$$

for some norm $\|\cdot\|$ stronger than the norm of $L^{2}\left(\Lambda \times \Omega_{\Lambda}, z d x d \mu_{\Lambda}^{\eta}\left(\omega_{\Lambda}\right)\right.$ ), we will get immediately (since $D_{x}^{+} H_{\Lambda}^{\eta}\left(\omega_{\Lambda}\right) \geqslant-2 B$ by $(\mathrm{C} 1)$ or $\left.(\mathrm{C} 2)\right)$,

$$
\mu_{\Lambda}^{\eta}\left(P_{t}^{\Lambda, \eta} F, P_{t}^{\Lambda, \eta} F\right)=-\int_{t}^{+\infty} \frac{d}{d s} \mu_{\Lambda}^{\eta}\left(P_{s}^{\Lambda, \eta} F, P_{s}^{\Lambda, \eta} F\right) d s \leqslant C(F) e^{-2 c t}, \quad \forall F \in \mathcal{D}
$$

for some constant $C(F)>0$ depending on $F$. This implies, by the spectral decomposition, $\lambda_{1} \geqslant c$ (true but not trivial).

For showing (2.14) which is of independent interest, as vaguely said in the introduction, we shall approximate the Poisson measure $P_{\Lambda}$ by the product Bernoulli measure on $\{0,1\}^{I_{N}}, \mu_{\Lambda}^{\eta}$ by Gibbs measures $\mu_{N}$ on $\{0,1\}^{I_{N}}$, $\mathcal{L}_{\Lambda}^{\eta}$ by $\mathcal{L}_{N}$ on some good test functions space $\mathcal{D}$ (this is possible if $\varphi$ is continuous and of finite range). A first questions arises:

1) to prove that $P_{t}^{N}=e^{t \mathcal{L}_{N}}$ verifies a relation similar to (2.14) with a constant $c>0$ independent of $N$ and with $D_{x}^{+}$substituted by some difference operator $\nabla$. This is provided by the Ligget $M-\varepsilon$ theorem which says roughly

$$
\left|\nabla P_{t}^{N} f\right| \leqslant e^{-t} e^{t \Gamma_{N}}|\nabla f|
$$

with the Ligget's matrix $\Gamma_{N}$. It remains to bound $\Gamma_{N}$ in some nice norm in such a way that is independent of $N$, and fortunately this works w.r.t. $\|\nabla f\|_{\infty}$ under (C1) or (C2). 
If the story stopped here, it would be simple and lucky. The real story is:

2) to transfer the Ligget's estimate on $\left(P_{t}^{N}\right)$ to $P_{t}^{\Lambda, \eta}$, though $\mathcal{L}_{N} \rightarrow \mathcal{L}_{\Lambda}^{\eta}$ on $\mathcal{D}$, we should prove that $\nabla P_{t}^{N} \rightarrow$ $D^{+} P_{t}^{\Lambda, \eta}$, or $P_{t}^{N} f_{n} \rightarrow P_{t}^{\Lambda, \eta} F$ in the sup norm. For the last convergence we should prove at first that $(\mathcal{L}, \mathcal{D})$ generates a unique semigroup on $b \mathcal{F}_{\Lambda}$ for applying the Trotter type theorem. The latter is quite difficult, for $\left(P_{t}^{\Lambda, \eta}\right)$ is not defined everywhere on $\Omega_{\Lambda}$ and even so, it is not at all strongly continuous on $b \mathcal{F}_{\Lambda}$ and the usual Trotter theorem can not be applied (but the involved techniques work, fortunately).

That is exactly the task of the following section.

\section{Uniqueness and the Markov process generated by $\mathcal{L}_{\Lambda}^{\eta}$}

Throughout this paper, for a pair of measure-function $(v, f), v(f):=\int f d v$. From now on, the bounded open (non-empty) domain $\Lambda$ and the boundary condition $\eta \in \Omega^{\varphi}$, though arbitrary, will be fixed. A genetic element of $\Omega_{\Lambda}$ will be often denoted by $\omega$ for simplicity of notation.

In this section we assume the stability (2.1), but not the regularity (2.2). The following duality relation ([16], Remarque 1, p. 518) will be used:

$$
\mathbb{E}^{P_{\Lambda}} \int_{\Lambda} F\left(\varepsilon_{x}^{+} \omega\right) G(x, \omega) z d x=\mathbb{E}^{P_{\Lambda}} F(\omega) \int_{\Lambda} G\left(x, \varepsilon_{x}^{-} \omega\right) d \omega(x)
$$

for all $\mathcal{F}_{\Lambda^{-}}$-measurable $F: \Omega_{\Lambda} \rightarrow \mathbb{R}^{+}$and all $\mathcal{B}(\Lambda) \otimes \mathcal{F}_{\Lambda}$-measurable $G: \Lambda \times \Omega_{\Lambda} \rightarrow \mathbb{R}^{+}$, where $\mathcal{B}(\Lambda)$ is the Borel $\sigma$-field of $\Lambda$.

\subsection{Uniqueness problem in $L^{p}\left(\mu_{\Lambda}^{\eta}\right)$}

Proposition 3.1. Assume the stability condition (2.1). Let

$$
\begin{aligned}
& \mathcal{D}_{1}:=\left\{f\left(\omega_{\Lambda}\left(A_{1}\right), \ldots, \omega_{\Lambda}\left(A_{n}\right)\right) 1_{\left.\max _{i} \omega_{\Lambda}\left(A_{i}\right) \leqslant 1 \mid n \geqslant 1, A_{i} \in \mathcal{A}, f: \mathbb{N}^{n} \rightarrow \mathbb{R} \text { bounded }\right\} ;}\right. \\
& \mathcal{D}_{2}:=\left\{F\left(\omega_{\Lambda}\left(h_{1}\right), \ldots, \omega_{\Lambda}\left(h_{n}\right)\right) ; h_{n} \in C_{b}^{\infty}(\Lambda), F \in C_{0}^{\infty}\left(\mathbb{R}^{n}\right), n \geqslant 1\right\} ;
\end{aligned}
$$

where $\mathcal{A}$ is an arbitrary subalgebra of $\mathcal{B}(\Lambda)$ such that $\sigma(\mathcal{A})=\mathcal{B}(\Lambda)$.

(a) For any $1 \leqslant p<+\infty, \mathcal{D}_{1}$ and $\mathcal{D}_{2}$ are both a core for the generator $\left(\mathcal{L}_{\Lambda}^{\eta}, \mathbb{D}_{p}\left(\mathcal{L}_{\Lambda}^{\eta}\right)\right)$ of $\left(P_{t}^{\Lambda, \eta}\right)$ on $L^{p}\left(\mu_{\Lambda}^{\eta}\right)$. In particular $\left(P_{t}^{\Lambda, \eta}\right)$ is the unique strongly continuous semigroup of bounded operators on $L^{p}\left(\mu_{\Lambda}^{\eta}\right)$ such that its generator extends $\left(\mathcal{L}_{\Lambda}^{\eta}, \mathcal{D}_{1}\right)$ or $\left(\mathcal{L}_{\Lambda}^{\eta}, \mathcal{D}_{2}\right)$; and $\left(\mathcal{L}_{\Lambda}^{\eta}, \mathcal{D}_{i}\right), i=1,2$, are essentially self-adjoint in $L^{2}\left(\mu_{\Lambda}^{\eta}\right)$.

(b) Given $F \in r \mathcal{F}_{\Lambda} \bigcap L^{2}\left(\mu_{\Lambda}^{\eta}\right)$. Then $F \in \mathbb{D}\left(\mathcal{E}_{\Lambda}^{\eta}\right)$ iff

$$
\int_{\Omega_{\Lambda}} d \mu_{\Lambda}^{\eta}\left(\omega_{\Lambda}\right) \int_{\Lambda} e^{-\beta D_{x}^{+} H_{\Lambda}^{\eta}\left(\omega_{\Lambda}\right)}\left(D_{x}^{+} F\right)^{2}\left(\omega_{\Lambda}\right) z d x<+\infty,
$$

and iff

$$
\int_{\Omega_{\Lambda}} d \mu_{\Lambda}^{\eta}\left(\omega_{\Lambda}\right) \sum_{x \in \operatorname{supp} \omega_{\Lambda}}\left(D_{x}^{-} F\right)^{2}\left(\omega_{\Lambda}\right)<+\infty .
$$

In those cases, the two quantities above coincide with $\mathcal{E}_{\Lambda}^{\eta}(F, F)$. 
Proof. (a) At first the second claim in (a) is a consequence of the first by [22] (Proposition 1.1), and the third claim is another expression of the first for $p=2$. To show the first claim, notice that $b \mathcal{F}_{\Lambda}$, identified with $L^{\infty}\left(\mu_{\Lambda}^{\eta}\right)$ up to $\mu_{\Lambda}^{\eta}$-equivalence, is a core for the generator of the Markov semigroup $\left(P_{t}^{\Lambda, \eta}\right)$ in $L^{p}\left(\mu_{\Lambda}^{\eta}\right)$ (true for any strongly continuous Markov semigroup). Hence it is enough to show that any $F \in b \mathcal{F}_{\Lambda}$ can be approximated by $F_{n} \in \mathcal{D}_{i}$ in the graph norm topology of $\mathcal{L}_{\Lambda}^{\eta}$ in $L^{p}\left(\mu_{\Lambda}^{\eta}\right)$, for $i=1,2$.

In fact we can find $F_{n} \in \mathcal{D}_{i}$ such that $F_{n} \rightarrow F, \mu_{\Lambda}^{\eta}$-a.s. and $\left|F_{n}\right| \leqslant\|F\|_{u}=\sup _{\omega \in \Omega_{\Lambda}}|F(\omega)|$ everywhere over $\Omega_{\Lambda}$. Then $\left\|F_{n}-F\right\|_{p}:=\left\|F_{n}-F\right\|_{L^{p}\left(\mu_{\Lambda}^{\eta}\right)} \rightarrow 0$ by the dominated convergence. It remains to prove that $\left\|\mathcal{L}_{\Lambda}^{\eta}\left(F_{n}-F\right)\right\|_{p} \rightarrow 0$. By the expression (2.8) and (2.9), we have

$$
\begin{aligned}
\left|\mathcal{L}_{\Lambda}^{\eta}\left(F_{n}-F\right)\right|(\omega) \leqslant & \left|\int_{\Lambda}\left[\left(F_{n}-F\right)(\omega)-\left(F_{n}-F\right)\left(\varepsilon_{x}^{-} \omega\right)\right] \omega(d x)\right|+z\left|\int_{\Lambda} e^{-\beta D_{x}^{+} H_{\Lambda}^{\eta}(\omega)} D_{x}^{+}\left(F_{n}-F\right)(\omega) d x\right| \\
\leqslant & \left|F_{n}-F\right|(\omega) \cdot N_{\Lambda}(\omega)+\int_{\Lambda}\left|F_{n}-F\right|\left(\varepsilon_{x}^{-} \omega\right) \omega(d x) \\
& +e^{2 B \beta N_{\Lambda}(\omega)} \int_{\Lambda} e^{-\beta h^{\eta}(x)} z d x \cdot\left|F_{n}-F\right|+e^{2 B \beta N_{\Lambda}(\omega)} \int_{\Lambda} e^{-\beta h^{\eta}(x)}\left|F_{n}-F\right|\left(\varepsilon_{x}^{+} \omega\right) z d x .
\end{aligned}
$$

Let us show that the four terms in the last sum converge all to 0 in $L^{p}\left(\mu_{\Lambda}^{\eta}\right)$, or in probability $\mu_{\Lambda}^{\eta} \sim P_{\Lambda}$ by the dominated convergence (by relation (2.6)).

The first and third terms pose no problem. For the second and fourth terms, it is enough to notice that by the duality formula (3.1), we have

$$
\begin{aligned}
& \int d P_{\Lambda}(\omega) \int_{\Lambda}\left|F_{n}-F\right|\left(\varepsilon_{x}^{-} \omega\right) \omega(d x)=z \int d P_{\Lambda}(\omega)\left|F_{n}-F\right| \cdot|\Lambda| \rightarrow 0 \\
& z \int d P_{\Lambda}(\omega) \int_{\Lambda} e^{-\beta h^{\eta}(x)}\left|F_{n}-F\right|\left(\varepsilon_{x}^{+} \omega\right) d x=\int d P_{\Lambda}(\omega)\left|F_{n}-F\right|(\omega) \int_{\Lambda} e^{-\beta h^{\eta}(x)} d \omega(x) \rightarrow 0 .
\end{aligned}
$$

(b) We shall prove only the first "iff" and the second can be proved in the same way. Given $F \in r \mathcal{F}_{\Lambda} \cap L^{2}\left(\mu_{\Lambda}^{\eta}\right)$ satisfying (3.3) and integer $L \geqslant 1$, let $F_{L}:=(F \wedge L) \vee(-L) \in b \mathcal{F}_{\Lambda} \subset \mathbb{D}\left(\mathcal{E}_{\Lambda}^{\eta}\right)$. Then $\left(D_{x}^{+} F_{L}\right)^{2}(\omega) \leqslant\left(D_{x}^{+} F\right)^{2}(\omega)$ and $\left(D_{x}^{+} F_{L}\right)^{2}(\omega) \uparrow\left(D_{x}^{+} F\right)^{2}(\omega)$ as $L \uparrow \infty$, for all $(x, \omega) \in \Lambda \times \Omega_{\Lambda}$. Then by the expression (2.10) and monotone convergence, $\mathcal{E}_{\Lambda}^{\eta}\left(F_{L}-F_{M}, F_{L}-F_{M}\right) \rightarrow 0$ as $L, M \rightarrow \infty$ and

$$
\mathcal{E}_{\Lambda}^{\eta}\left(F_{L}, F_{L}\right) \rightarrow \int_{\Omega_{\Lambda}} d \mu_{\Lambda}^{\eta} \int_{\Lambda} e^{-\beta D_{x}^{+} H_{\Lambda}^{\eta}\left(\omega_{\Lambda}\right)}\left(D_{x}^{+} F\right)^{2}\left(\omega_{\Lambda}\right) z d x<+\infty,
$$

where it follows that $F \in \mathbb{D}\left(\mathcal{E}_{\Lambda}^{\eta}\right)$ and the last quantity coincides with $\mathcal{E}_{\Lambda}^{\eta}(F, F)$.

Inversely let $F \in \mathbb{D}\left(\mathcal{E}_{\Lambda}^{\eta}\right)$. Hence by definition there exists a sequence $\left(F_{n}\right)_{n} \geqslant 1 \subset b \mathcal{F}_{\Lambda}$ such that $F_{n} \rightarrow F$ in $L^{2}\left(\mu_{\Lambda}^{\eta}\right)$ and $\mathcal{E}_{\Lambda}^{\eta}\left(F_{n}-F, F_{n}-F\right) \rightarrow 0$ as $n \rightarrow \infty$. By taking a subsequence and a re-definition of $F_{n}, F$ if necessary, we may assume without loss of generality that $F_{n}(\omega) \rightarrow F(\omega)$ everywhere in $\Omega_{\Lambda}$. Hence $D_{x}^{+} F_{n}(\omega) \rightarrow D_{x}^{+} F(\omega)$ for all $(x, \omega) \in \Lambda \times \Omega_{\Lambda}$. Thus by the expression (2.10) of $\mathcal{E}_{\Lambda}^{\eta}\left(F_{n}, F_{n}\right)$, we see that

$$
\mathcal{E}_{\Lambda}^{\eta}\left(F_{n}, F_{n}\right) \rightarrow \int_{\Omega_{\Lambda}} d \mu_{\Lambda}^{\eta} \int_{\Lambda} e^{-\beta D_{x}^{+} H_{\Lambda}^{\eta}\left(\omega_{\Lambda}\right)}\left(D_{x}^{+} F\right)^{2}\left(\omega_{\Lambda}\right) z d x
$$

the desired result. 


\subsection{Construction of the associated Markov process}

As probabilists we always want to construct a good Markov process associated with generator $\mathcal{L}_{\Lambda}^{\eta}$. This was done by Holley and Stroock [7] and Picard [16]. Unfortunately the conditions imposed in those known works are not all verified for the model used here.

Proposition 3.2. Assume the stability condition (2.1). There is a strong Markov process $\left(\left(X_{t}\right)_{t} \geqslant 0,\left(\mathbb{P}_{\omega}\right)_{\omega \in \Omega_{\Lambda}}\right)$ valued in $\Omega_{\Lambda} \cup\{\partial\}$ where $\Omega_{\Lambda}$ is equipped with the weak convergence topology and $\partial$ is an extra isolated point to $\Omega_{\Lambda}$, defined on some measurable space $(W, \mathcal{B})$, such that

(i) For any $\omega \in \Omega_{\Lambda}, \mathbb{P}_{\omega}$-a.s., $X_{0}=\omega, X_{t}=\partial(\forall t \geqslant \zeta), t \rightarrow X_{t}$ is right continuous and of pure jumps type on $[0, \zeta)$, i.e., there are a sequence of stopping times $0=T_{0}<T_{1}<T_{2}<\cdots<T_{n} \uparrow \zeta$ (w.r.t. the natural filtration of $\left(X_{t}\right)$ ) such that $X_{t}=X_{T_{n}}$ for all $t \in\left[T_{n}, T_{n+1}\right)$.

(ii) Let $P_{t} F(\omega):=\mathbb{E}^{\omega}\left(F\left(X_{t}\right) 1_{t<\zeta}\right):=\mathbb{E}^{\mathbb{P}_{\omega}}\left(F\left(X_{t}\right) 1_{t<\zeta}\right)$. It is a semigroup of transition kernels on b $\mathcal{F}_{\Lambda}$ and for any $F \in b \mathcal{F}_{\Lambda}$ and any $\omega \in \Omega_{\Lambda}, P_{t} F(\omega)$ is continuous differentiable on $\mathbb{R}^{+}$and

$$
\frac{d}{d t} P_{t} F(\omega)=\left(\mathcal{L}_{\Lambda}^{\eta} P_{t} F\right)(\omega) .
$$

(iii) If $v$ is a nonnegative measure on $\Omega_{\Lambda}$ such that $v\left(\left[N_{\Lambda} \leqslant L\right]\right)<+\infty, \forall L \in \mathbb{N}$ and $v\left(\mathcal{L}_{\Lambda}^{\eta} F\right)=0, \forall F \in \mathcal{D}_{1}(c f$. (3.2)), then $v=C \mu_{\Lambda}^{\eta}$ for some constant $C$.

(iv) For $\mu_{\Lambda}^{\eta}$-a.s. $\omega \in \Omega_{\Lambda}, \mathbb{P}_{\omega}(\zeta=+\infty)=1$ and $P_{t} F(\omega)=P_{t}^{\Lambda, \eta} F(\omega), \mu_{\Lambda}^{\eta}$-a.s. for all $t \geqslant 0, F \in b \mathcal{F}_{\Lambda}$.

If we suppose moreover that there are constants $K_{1}, K_{2} \geqslant 0$ (depending eventually on $\Lambda$ ) such that,

$$
z \int_{\Lambda} e^{-\beta \sum_{i=1}^{n} \varphi\left(x_{i}-x\right)} d x \leqslant K_{1} n+K_{2}, \quad \forall n \geqslant 1, \forall x_{1}, \ldots, x_{n} \in \Lambda
$$

then $\mathbb{P}_{\omega}(\zeta=+\infty)=1, \forall \omega \in \Omega_{\Lambda}$, and for any $F \in b \mathcal{F}_{\Lambda}$ and any $\omega \in \Omega_{\Lambda}, P_{t} \mathcal{L}_{\Lambda}^{\eta} F(\omega)$ is finite and continuous on $t$ and

$$
\frac{d}{d t} P_{t} F(\omega)=P_{t}\left(\mathcal{L}_{\Lambda}^{\eta} F\right)(\omega)
$$

moreover if $\left(\tilde{P}_{t}(\omega, \cdot)_{t} \geqslant 0\right.$ is any semigroup of kernels on $b \mathcal{F}_{\Lambda}$ such that the Kolmogorov equation (3.5) holds for all $F \in \mathcal{D}_{i}(c f .(3.2))$ and $\sup _{\|F\|_{u} \leqslant 1}\left\|\tilde{P}_{t} F\right\|_{u}$ is bounded on any bounded interval $t \in[0, T]$, then $\tilde{P}_{t}=P_{t}$; where $i=1$ or 2 .

Remarks 3.3. The last uniqueness allows us to say, without ambiguity, that $\left(P_{t}\right)$ is the semigroup of kernels on $b \mathcal{F}_{\Lambda}$ generated by $\mathcal{L}_{\Lambda}^{\eta}$. This result satisfies not only our probabilistic desire, but it is also technically crucial in the approximation procedure in the proof of our main results. Notice that this Markov process is constructed for every starting point. It is an easy application of the theory of jumps processes.

Let us also compare (3.5) and (3.7): they are usually known as to be equivalent for strongly continuous semigroup for $F$ in domain of generator. But on the bad space " $b \mathcal{F}_{\Lambda}$ ", $\left(P_{t}\right)$ is not strongly continuous and the domain of its generator has many different definitions. In our case, (3.7) is stronger than (3.5). Moreover (3.7) implies both $\mathbb{P}_{\omega}(\zeta=+\infty)=P_{t} 1(\omega)=1, \forall \omega$ (the non-explosion) and the last uniqueness, but (3.5) does not.

The linear growth condition (3.6) about the birth rate is adopted from [16], Proposition 4 where another condition, which is not satisfied here, is imposed too. It is (fortunately) satisfied for a family of important interaction functions such as those verifying $(\mathrm{C} 1)$ or hard core condition. But we believe that the conservability (for any starting point) should be true under the only stability condition. 
Proof. Step 1. The construction in this step is well known (e.g. [8]). For any $(\omega, A) \in \Omega_{\Lambda} \times \mathcal{F}_{\Lambda}$, let

$$
\begin{aligned}
& b(\omega):=z \int_{\Lambda} e^{-\beta D_{x}^{+} H_{\Lambda}^{\eta}(\omega)} d x \quad \text { (birth rate), } \\
& c(\omega):=N_{\Lambda}(\omega)+b(\omega) \quad \text { (total intensity), } \\
& Q(\omega, A):=\frac{\int_{\Lambda} 1_{A}\left(\varepsilon_{x}^{-} \omega\right) \omega(d x)+z \int_{\Lambda} e^{-\beta D_{x}^{+} H_{\Lambda}^{\eta}(\omega)} 1_{A}\left(\varepsilon_{x}^{+} \omega\right) d x}{c(\omega)}
\end{aligned}
$$

(note that $b(\mathbf{0})=c(\mathbf{0})=z|\Lambda|>0$ and then $c(\omega)>0$ over $\left.\Omega_{\Lambda}\right) . Q$ is a Markov kernel on $\left(\Omega_{\Lambda}, \mathcal{F}_{\Lambda}\right)(Q 1(\omega)=$ $1, \forall \omega)$. Let $\mathbb{Q}_{\omega}$ be the probability measure on $\Omega_{\Lambda}^{\mathbb{N}}$ such that its coordinates system $\left(Y_{n}\right)$ is a Markov chain with transition kernel $Q$ and with the starting point $Y_{0}=\omega, \mathbb{Q}_{\omega}$-a.s. and $\gamma$ be the exponential law of parameter 1 on $\mathbb{R}^{+}$. Consider the product space $\left(W:=\Omega_{\Lambda}^{\mathbb{N}} \times\left(\mathbb{R}^{+}\right)^{\mathbb{N}}, \mathcal{B}=\mathcal{F}_{\Lambda}^{\otimes \mathbb{N}} \otimes \mathcal{B}\left(\mathbb{R}^{+}\right)^{\otimes \mathbb{N}}, \mathbb{P}_{\omega}=\mathbb{Q}_{\omega} \otimes \gamma^{\mathbb{N}}\right)$ whose coordinates system will be denoted by $\left(Y_{n}, \gamma_{n}\right)_{n} \geqslant 0$. Set

$$
\begin{aligned}
& T_{0}=0, \quad T_{n}:=\sum_{k=1}^{n} \frac{\gamma_{k}}{c\left(Y_{k-1}\right)}, \quad \forall n \geqslant 1 ; \\
& X_{t}:=Y_{n}, \quad \forall t \in\left[T_{n}, T_{n+1}\right), \quad \forall n \in \mathbb{N} ; \\
& X_{t}:=\partial, \quad \forall t \geqslant \zeta:=\sup _{n \geqslant 1} T_{n}=\sum_{k=1}^{\infty} \frac{\gamma_{k}}{c\left(Y_{k-1}\right)}
\end{aligned}
$$

where $\partial$ is an extra point to $\Omega_{\Lambda}$. $\left(\left(X_{t}\right), \mathbb{P}_{\omega}\right)$ constitutes a strong Markov process valued in $\Omega_{\Lambda} \cup\{\partial\}$, satisfying (i).

Step 2. Let us now verify (ii), (iii) and (iv). At first (ii) follows from [8], Theorem 10.24 (though it is stated for conservative pure jumps processes, but the same proof works in the eventual non-conservative case here). For (iii), notice that $\forall F \in \mathcal{D}_{1}$ nonnegative, $F \in L^{1}(c(\omega) d \nu(\omega))$ and $\mathcal{L}_{\Lambda}^{\eta} F \in b \mathcal{F}_{\Lambda} \cap L^{1}(v)$, and

$$
\int(Q F-F)(\omega) c(\omega) d v(\omega)=\int \mathcal{L}_{\Lambda}^{\eta} F(\omega) d v(\omega)
$$

Hence $v$ verifies $v\left(\mathcal{L}_{\Lambda}^{\eta} F\right)=0, \forall 0 \leqslant F \in \mathcal{D}_{1}$ iff $c(\omega) d v(\omega)$ is an invariant measure of $Q$. As $Q^{k}(\omega, \mathbf{0})>0$, where $k=N_{\Lambda}(\omega), Q$ is irreducible in the classical language of Markov chains; moreover since $\mu_{\Lambda}^{\eta}\left(\mathcal{L}_{\Lambda}^{\eta} F\right)=0, \forall F \in$ $b \mathcal{F}_{\Lambda}$ by (2.10), then $c(\omega) d \mu_{\Lambda}^{\eta}(\omega)$ is the only invariant measure of $Q$, which yields (iii) by the strict positivity of $c(\omega)$ over $\Omega_{\Lambda}$.

For (iv), notice at first that

$$
\{\zeta=+\infty\}=\left\{\sum_{k=1}^{\infty} \frac{1}{c\left(Y_{k-1}\right)}=+\infty\right\}, \quad \mathbb{P}_{\omega} \text {-a.s. }
$$

Since $c(\omega) d \mu_{\Lambda}^{\eta}(\omega)$ is an finite invariant measure of $Q$, then $Y=\left(Y_{n}\right)$ is positively recurrent. In particular $\mathbb{P}_{\omega}\left(Y_{n}=\mathbf{0}\right.$, i.o. $)=1$ for $\mu_{\Lambda}^{\eta}$-a.e. $\omega$ (i.o. $=$ infinitely often). Thus $\mathbb{P}_{\omega}(\zeta=+\infty)=1$ for $\mu_{\Lambda}^{\eta}$-a.e. $\omega$.

For all $F \in b \mathcal{F}_{\Lambda}$, by (ii) and the fact that $\mu_{\Lambda}^{\eta}\left(\mathcal{L}_{\Lambda}^{\eta} P_{t} F\right)=0$, we see that $\mu_{\Lambda}^{\eta}\left(P_{t} F\right)=\mu_{\Lambda}^{\eta}(F), \forall t \geqslant 0$. Then $\left(P_{t}\right)$ is a strongly continuous semigroup of contractions on $L^{2}\left(\mu_{\Lambda}^{\eta}\right)$ whose generator coincides with $\mathcal{L}_{\Lambda}^{\eta}$ on $b \mathcal{F}_{\Lambda}$. By the uniqueness result in Proposition 3.1, $P_{t} F=P_{t}^{\Lambda, \eta} F, \mu_{\Lambda}^{\eta}$-a.s.

Step 3. Let us prove the conservability and (3.7) under the extra condition (3.6), which is one of conditions in [16], Proposition 4. Fix our starting point $\omega \in \Omega_{\Lambda}$.

By (3.8) and the fact that $c\left(Y_{k}\right) \geqslant N_{\Lambda}\left(Y_{k}\right)$, we have $\mathbb{P}_{\omega}\left(\zeta=+\infty / N_{\Lambda}\left(Y_{n}\right) \leqslant L\right.$, i.o. $)=1$, for any $L>0$. Consequently on $[\zeta<+\infty]$, we have $\mathbb{P}_{\omega}$-a.s.,

$$
\lim _{t \rightarrow \zeta-} N_{\Lambda}\left(X_{t}\right)=\lim _{n \rightarrow \infty} N_{\Lambda}\left(Y_{n}\right)=+\infty
$$


(i.e. the life time $\zeta$, if finite, coincides with the explosion time in number of particles). To control $N_{\Lambda}\left(X_{t}\right)$, notice that for any $F \in r \mathcal{F}_{\Lambda}$ such that $Q^{k}(\omega,|F|)<+\infty, \forall k \geqslant 1$,

$$
F\left(X_{t \wedge T_{n}}\right)-F(\omega)-\int_{0}^{t \wedge T_{n}} \mathcal{L}_{\Lambda}^{\eta} F\left(X_{s}\right) d s
$$

is a $\mathbb{P}_{\omega}$-martingale for each $n$. Applying it to $F(\omega)=N_{\Lambda}(\omega)$, and noting that by condition (3.6)

$$
\begin{aligned}
\mathcal{L}_{\Lambda}^{\eta} N_{\Lambda}(\omega) & =-N_{\Lambda}(\omega)+z \int_{\Lambda} e^{-\beta D_{x}^{+} H_{\Lambda}^{\eta}(\omega)} d x \\
& \leqslant\left(K_{1} e^{-\beta \inf _{x \in \Lambda} h^{\eta}(x)}-1\right) N_{\Lambda}(\omega)+K_{2} e^{-\beta \inf _{x \in \Lambda} h^{\eta}(x)} \leqslant L\left(N_{\Lambda}(\omega)+1\right)
\end{aligned}
$$

for some constant $L$, we see that

$$
N_{\Lambda}\left(X_{t \wedge T_{n}}\right)-N_{\Lambda}(\omega)-L \int_{0}^{t}\left[1+N_{\Lambda}\left(X_{s \wedge T_{n}}\right)\right] d s
$$

is a supermartingale. Thus $\mathbb{E}^{\omega} N_{\Lambda}\left(X_{t \wedge T_{n}}\right) \leqslant e^{L t}\left(N_{\Lambda}(\omega)+1-e^{-L t}\right)$ by Gronwall inequality. By the fact that $[\zeta<+\infty] \subset\left[\lim _{t \uparrow \zeta} N_{\Lambda}\left(X_{t}\right)=+\infty\right]$ shown previously, we get by Fatou's lemma that for each $T>0$,

$$
+\infty \cdot \mathbb{P}_{\omega}(\zeta \leqslant T)=\mathbb{E}^{\omega} \lim _{t \uparrow \zeta, n \rightarrow \infty} N_{\Lambda}\left(X_{t \wedge T_{n}}\right) 1_{\zeta \leqslant T} \leqslant e^{L T}\left(N_{\Lambda}(\omega)+1\right)
$$

where it follows the desired non-explosion: $\mathbb{P}_{\omega}(\zeta<+\infty)=0$. Let us show now

$$
\mathbb{E}^{\omega} \sup _{t \in[0, T]} N_{\Lambda}\left(X_{t}\right)<+\infty, \quad \forall T>0 .
$$

In fact, consider the local martingale $M_{t}:=N_{\Lambda}\left(X_{t}\right)-N_{\Lambda}(\omega)-\int_{0}^{t} \mathcal{L}_{\Lambda}^{\eta} N_{\Lambda}\left(X_{S}\right) d s$. Its predictable quadratic process is given by

$$
\langle M\rangle_{t}=\int_{0}^{t}\left(N_{\Lambda}+z \int_{\Lambda} e^{-\beta D_{x}^{+} H_{\Lambda}^{\eta}} d x\right)\left(X_{s}\right) d s \leqslant L \int_{0}^{t}\left(N_{\Lambda}\left(X_{s}\right)+1\right) d s
$$

which is $\mathbb{P}_{\omega}$-integrable by the estimation above. Hence $M_{t}$ is a true martingale in $L^{2}\left(\mathbb{P}_{\omega}\right)$. Now the desired (3.9) follows by the maximal inequality of Doob.

Having (3.9) we can conclude our proof of (3.7) easily. For every $F \in b \mathcal{F}_{\Lambda}$, since

$$
\left|\mathcal{L}_{\Lambda}^{\eta} F(\omega)\right| \leqslant 2\|F\|_{u} L\left[N_{\Lambda}(\omega)+1\right]
$$

for some constant $L$ by (3.6), then $P_{t}\left|\mathcal{L}_{\Lambda}^{\eta} F\right|(\omega)<+\infty$ for all $\omega$. Moreover for each $t$ fixed, since $X_{s}=X_{t}, \mathbb{P}_{\omega}$-a.s. for all $s$ sufficiently close to $t$, we then have for all $G \in r \mathcal{F}_{\Lambda}$ satisfying $|G| \leqslant L\left(N_{\Lambda}+1\right)$,

$$
\lim _{s \rightarrow t} P_{s} G(\omega)=\lim _{s \rightarrow t} \mathbb{E}^{\omega} G\left(X_{s}\right)=\mathbb{E}^{\omega} G\left(X_{t}\right)=P_{t} G(\omega)
$$

by dominated convergence and (3.9). Consequently $P_{t} \mathcal{L}_{\Lambda}^{\eta} F(\omega)$ is continuous on $t$.

Moreover (3.9) implies that the local martingale $F\left(X_{t}\right)-F(\omega)-\int_{0}^{t} \mathcal{L}_{\Lambda}^{\eta} F\left(X_{s}\right) d s$ is a true martingale. Finally taking expectation in this martingale we get

$$
P_{t} F(\omega)-F(\omega)=\int_{0}^{t} P_{s} \mathcal{L}_{\Lambda}^{\eta} F(\omega) d s, \quad \forall \omega \in \Omega_{\Lambda}, t \geqslant 0,
$$

where (3.7) follows. 
Step 4. It remains to show the last uniqueness. Notice that if $\tilde{P}_{t} F=P_{t} F$ for all $F \in \mathcal{D}_{1}$, then they coincide on $b \mathcal{F}_{\Lambda}$ (since $\tilde{P}_{t}$ is assumed to be kernel as $P_{t}$ ). Then it is enough to show that for any $F \in \mathcal{D}_{1}, s \rightarrow P_{s} \tilde{P}_{t-s} F(\omega)$ is continuous on $[0, t]$ and

$$
\frac{d}{d s^{+}} P_{s} \tilde{P}_{t-s} F(\omega)=0, \quad \forall s \in(0, t) .
$$

We begin with the following fact: if $G_{\varepsilon} \rightarrow G$ everywhere (as $\left.\varepsilon \rightarrow 0\right)$ and if $\left|G_{\varepsilon}\right| \leqslant L\left(N_{\Lambda}+1\right)$, then

$$
P_{s+\varepsilon} G_{\varepsilon}(\omega) \rightarrow P_{S} G(\omega), \quad \forall \omega .
$$

In fact, for all $\varepsilon$ sufficiently small, $X_{s+\varepsilon}=X_{s}$, a.s. and then as $\varepsilon \rightarrow 0$,

$$
G_{\varepsilon}\left(X_{s+\varepsilon}\right) \rightarrow G\left(X_{s}\right), \quad \mathbb{P}_{\omega} \text {-a.s. }
$$

By (3.9), we so have $P_{s+\varepsilon} G_{\varepsilon}(\omega)=\mathbb{E}^{\omega} G_{\varepsilon}\left(X_{s+\varepsilon}\right) \rightarrow \mathbb{E}^{\omega} G\left(X_{s}\right)=P_{s} G(\omega)$.

The previous fact gives the continuity of $s \rightarrow P_{s} \tilde{P}_{t-s} F$ on $[0, t]$. For any $s \in(0, t)$,

$$
\begin{aligned}
\frac{d}{d s^{+}} P_{s} \tilde{P}_{t-s} F(\omega) & =\lim _{\varepsilon \rightarrow 0+} \frac{P_{s+\varepsilon} \tilde{P}_{t-s-\varepsilon} F-P_{s} \tilde{P}_{t-s} F}{\varepsilon} \\
& =\lim _{\varepsilon \rightarrow 0+} P_{s+\varepsilon} \frac{\tilde{P}_{t-s-\varepsilon} F-\tilde{P}_{t-s} F}{\varepsilon}+\lim _{\varepsilon \rightarrow 0+} \frac{P_{s+\varepsilon} \tilde{P}_{t-s} F-P_{s} \tilde{P}_{t-s} F}{\varepsilon} .
\end{aligned}
$$

The last limit above is $P_{s} \mathcal{L}_{\Lambda}^{\eta} \tilde{P}_{t-s} F$ by (3.7). For the first limit above, by the assumption,

$$
\frac{\tilde{P}_{t-s-\varepsilon} F-\tilde{P}_{t-s} F}{\varepsilon} \rightarrow-\mathcal{L}_{\Lambda}^{\eta} \tilde{P}_{t-s} F
$$

which is uniformly bounded by $\sup _{u \in[0, t]}\left|\mathcal{L}_{\Lambda}^{\eta} \tilde{P}_{u} F\right| \leqslant 2 \sup _{u \in[0, t]}\left\|\tilde{P}_{u} F\right\| L\left(1+N_{\Lambda}\right)$. Then the first limit above is $-P_{s} \mathcal{L}_{\Lambda}^{\eta} \tilde{P}_{t-s} F$, by (3.10). Thus $\frac{d}{d s^{+}} P_{S} \tilde{P}_{t-s} F(\omega)=0$ as required.

To illustrate the usefulness of the previous result, let us present a comparison result (of independent interest).

Corollary 3.4. Assume (C1) or (C2) in Theorem 2.1. Let

$$
\mathcal{L}_{\Lambda}^{0} F(\omega):=\sum_{x \in \operatorname{supp} \omega} D_{x}^{-} F(\omega)+z e^{2 B \beta} \int_{\Lambda} D_{x}^{+} F(\omega) d x, \quad \forall F \in b \mathcal{F}_{\Lambda}
$$

which generates a Markov semigroup $P_{t}^{0}$ on $b \mathcal{F}_{\Lambda}$ by Proposition 3.2. If $F \in b \mathcal{F}_{\Lambda}$ verifies $D_{x}^{+} F(\omega) \geqslant 0$, dx-a.e. for every $\omega \in \Omega_{\Lambda}$, then

$$
P_{t} F(\omega) \leqslant P_{t}^{0} F(\omega), \quad \forall \omega \in \Omega_{\Lambda}, t \geqslant 0 .
$$

In particular for all nonnegative $F_{1}, \ldots, F_{n} \in b \mathcal{F}_{\Lambda}$ such that $D_{x}^{+} F_{k} \geqslant 0$ dx-a.e. for every $\omega \in \Omega_{\Lambda}$ and $0 \leqslant t_{1}<$ $t_{2}<\cdots<t_{n}$, we have

$$
\mathbb{E}^{\mathbb{P} \omega} F_{1}\left(X_{t_{1}}\right) \cdots F_{n}\left(X_{t_{n}}\right) \leqslant \mathbb{E}^{\mathbb{P}_{\omega}^{0}} F_{1}\left(X_{t_{1}}^{0}\right) \cdots F_{n}\left(X_{t_{n}}^{0}\right)
$$

where $\left(\left(X_{t}^{0}\right)_{t \geqslant 0}, \mathbb{P}_{\omega}^{0}\right)$ is the Markov process with transition semigroup $\left(P_{t}^{0}\right)$ and starting point $\omega$.

Recall that the "free" semigroup $P_{t}^{0}$ is symmetric w.r.t. the Poisson measure on $\Lambda$ with intensity measure $e^{2 B \beta} z d x$, its spectral gap is 1 , and has an explicit expression by means of the chaos decomposition (cf. [21]). 
Proof. Let $F \in b \mathcal{F}_{\Lambda}, t>0$. By Proposition $3.2(3.7), P_{s} P_{t-s}^{0} F(\omega)$ is continuously differentiable on $[0, t]$ and

$$
\frac{d}{d s} P_{s} P_{t-s}^{0} F(\omega)=P_{s}\left(\mathcal{L}_{\Lambda}^{\eta}-\mathcal{L}^{0}\right) P_{t-s}^{0} F(\omega)=P_{s}\left(\int_{\Lambda}\left(e^{-\beta D_{x}^{+} H_{\Lambda}^{\eta}}-e^{2 B \beta}\right) D_{x}^{+} P_{t-s}^{0} F z d x\right)(\omega) .
$$

Since $\left[\mathcal{L}^{0}, D_{x}^{+}\right] F(\omega):=\left(\mathcal{L}^{0} D_{x}^{+}-D_{x}^{+} \mathcal{L}^{0}\right) F(\omega)=D_{x}^{+} F(\omega), \forall x \notin \operatorname{supp} \omega$ (a simple calculus), we have

$$
D_{x}^{+} P_{t}^{0} F(\omega)=e^{-t} P_{t}^{0} D_{x}^{+} F(\omega), \quad d x \text {-a.e., } \forall \omega \in \Omega_{\Lambda}, t \geqslant 0
$$

(this relation implies that the spectral gap of $-\mathcal{L}^{0}$ is 1$)$. Thus $D_{x}^{+} P_{t-s}^{0} F(\omega) \geqslant 0, d x$-a.e. for all $\omega$. On the other hand, since $D_{x}^{+} H_{\Lambda}^{\eta} \geqslant-2 B$ over $\Omega_{\Lambda}$ by (C1) or (C2), then $\frac{d}{d s} P_{s} P_{t-s}^{0} F(\omega) \leqslant 0$ for all $s \in[0, t]$. Thus $P_{t} F(\omega) \leqslant P_{t}^{0} F(\omega)$, the desired (3.12). The last inequality follows from (3.12) by iteration.

\section{Approximation of continuous gas by discrete spin systems}

Throughout this section we assume, besides the stability (2.1) and the regularity (2.2), moreover that $\varphi: \mathbb{R}^{d} \rightarrow$ $(-\infty,+\infty]$ is continuous and is of finite range, i.e., there exists some $r>0$ such that $\varphi(x)=0$ if $|x|>r$.

\subsection{Construction of the approximating discrete spin systems}

Consider a sequence $\left(\mathcal{P}^{N}:=\left\{A_{i}^{N} ; i \in I_{N}=\left\{1, \ldots, 2^{N}\right\}\right\}, N \geqslant 1\right)$ of partitions of $\Lambda$ (i.e., for each $N \geqslant 1$, $\Lambda=\bigcup_{i \in I_{N}} A_{i}^{N}$ and $\left(A_{i}^{N}, i \in I_{N}\right) \subset \mathcal{B}(\Lambda)$ disjoint $)$, such that

- the maximum $r(N)$ of the diameters of $A_{i}^{N}, i=1, \ldots, 2^{N}$, goes to 0 as $N$, goes to infinity;

- each $A_{i}^{N}$ is the union of two disjoint subsets $A_{j}^{N+1}, A_{j+1}^{N+1}$ in the $(N+1)$ th partition.

This sequence of partitions will be fixed as well as points $x_{i}^{N} \in A_{i}^{N}, i \in I_{N}, N \geqslant 1$. Then under the Poisson measure $P$,

$$
q_{N}(\omega)=\left(\omega\left(A_{1}^{N}\right) \wedge 1, \ldots, \omega\left(A_{2^{N}}^{N}\right) \wedge 1\right) \in\{0,1\}^{2^{N}}
$$

is a sequence of independent Bernoulli random variables respectively with laws $\left(\gamma_{i}^{N}\right)_{i \in I_{N}}$, where the success probability $\gamma_{i}^{N}(1)=1-e^{-\left|A_{i}^{N}\right| z} \sim z\left|A_{i}^{N}\right|, i \in I_{N}$. Moreover

$$
\Omega_{\Lambda}^{N}:=\left\{\omega \in \Omega_{\Lambda} ; \max _{i \in I_{N}} \omega\left(A_{i}^{N}\right) \leqslant 1\right\}
$$

increases to $\Omega_{\Lambda}$. Consequently $\forall \omega \in \Omega_{\Lambda}$,

$$
p_{N}(\omega):=\sum_{i \in I_{N}}\left(\omega\left(A_{i}^{N}\right) \wedge 1\right) \delta_{x_{i}^{N}} \rightarrow \omega
$$

weakly (as bounded measures on $\Lambda$ ).

Regarding the expression (2.8) of $\mathcal{L}_{\Lambda}^{\eta}$, we see that a good candidate for approximating $\mathcal{L}_{\Lambda}^{\eta}$ is the following generator for real function $f$ on $\{0,1\}^{I_{N}}=\{0,1\}^{2^{N}}$,

$$
\mathcal{L}_{N} f(\sigma)=\sum_{i \in I_{N}} D_{i}^{-} f(\sigma)+\sum_{i \in I_{N}}\left(e^{z\left|A_{i}^{N}\right|}-1\right) e^{-\beta D_{i}^{+} H^{N}(\sigma)} D_{i}^{+} f(\sigma), \quad \forall \sigma \in\{0,1\}^{I_{N}}
$$

where 


$$
\begin{aligned}
H^{N}(\sigma) & =\sum_{1 \leqslant i<j \leqslant 2^{N}, i \neq j} \varphi\left(x_{i}^{N}-x_{j}^{N}\right) \sigma(i) \sigma(j)+\sum_{i=1}^{2^{N}} \sum_{y \in \operatorname{supp}(\eta) \cap \Lambda^{c}} \varphi\left(x_{i}^{N}-y\right) \sigma(i) \\
& =H_{\Lambda}^{\eta}\left(\sum_{i=1}^{n} \sigma(i) \delta_{x_{i}^{N}}\right)
\end{aligned}
$$

and

$$
D_{i}^{-} f(\sigma):=f\left(\sigma^{i-}\right)-f(\sigma), \quad D_{i}^{+} f(\sigma):=f\left(\sigma^{i+}\right)-f(\sigma)
$$

and $\sigma^{i \pm}(j)=\sigma(j)$ for $j \neq i$ and $\sigma^{i+}(i)=1$ and $\sigma^{i-}(i)=0$. In fact we have

\section{Lemma 4.1.}

(a) For any $f:\{0,1\}^{I_{N}} \rightarrow \mathbb{R}$, let $F(\omega)=f\left(q_{N} \omega\right)$. Then for all $\omega \in \Omega_{\Lambda}$,

$$
\begin{aligned}
\left(\mathcal{L}_{N} f\right)\left(q_{N} \omega\right)= & \sum_{x \in \Lambda} D_{x}^{-} F(\omega)+\sum_{i \in I_{N}} 1_{\omega\left(A_{i}^{N}\right)>1}\left(D_{i}^{-} f\right)\left(q_{N} \omega\right) \\
& +\sum_{i \in I_{N}} \int_{A_{i}^{N}} \frac{e^{z\left|A_{i}^{N}\right|}-1}{\left|A_{i}^{N}\right|} e^{-\beta D_{x}^{+} H_{\Lambda}^{\eta}\left(p_{N} \omega\right)} D_{x}^{+} F(\omega) d x
\end{aligned}
$$

(b) Let

(warning: $\left.D_{x}^{+} H_{\Lambda}^{\eta}\left(p_{N} \omega\right) \neq\left(D_{x}^{+} H_{\Lambda}^{\eta}\right)\left(p_{N} \omega\right)\right)$, where $\frac{e^{z\left|A_{i}^{N}\right|}-1}{\left|A_{i}^{N}\right|}:=z$ if $\left|A_{i}^{N}\right|=0$.

$$
\mu_{N}(\sigma)=\frac{e^{-\beta H^{N}(\sigma)}}{C_{N}} \prod_{i \in I_{N}} \gamma_{i}^{N}(\sigma)
$$

be the Gibbs measure on $\{0,1\}^{2^{N}}$ associated with the Hamiltonian $H^{N}$ given in (4.2), where $\gamma_{i}^{N}$ is the Bernoulli measure such that $\gamma_{i}^{N}(1)=1-e^{-z\left|A_{i}^{N}\right|}$, and $C_{N}$ is the normalizing constant. Then $\mathcal{L}_{N}$ is symmetric on $L^{2}\left(\{0,1\}^{I_{N}}, \mu_{N}\right)$ and for all real functions $f, g$ on $\{0,1\}^{I_{N}}$,

$$
\begin{aligned}
\mathcal{E}_{N}(f, g) & :=\mu_{N}\left(g\left(-\mathcal{L}_{N} f\right)\right)=\mu_{N}\left(\sum_{i \in I_{N}} D_{i}^{-} f \cdot D_{i}^{-} g\right) \\
& =\mu_{N}\left(\sum_{i \in I_{N}}\left(e^{z\left|A_{i}^{N}\right|}-1\right) e^{-\beta D_{i}^{+} H^{N}} D_{i}^{+} f \cdot D_{i}^{+} g\right) .
\end{aligned}
$$

Proof. Part (a) is an immediate consequence of the following simple relations

$$
\begin{aligned}
& D_{x}^{+} f\left(q_{N} \omega\right)=\left(D_{i}^{+} f\right)\left(q_{N} \omega\right), \quad \forall x \in A_{i}^{N}, \forall \omega \in \Omega_{\Lambda}, \\
& D_{x}^{-} f\left(q_{N} \omega\right)=1_{\omega\left(A_{i}^{N}\right) \leqslant 1}\left(D_{i}^{-} f\right)\left(q_{N} \omega\right), \quad \forall x \in A_{i}^{N} \cap \operatorname{supp} \omega, \\
& H^{N}\left(q_{N} \omega\right)=H_{\Lambda}^{\eta}\left(p_{N} \omega\right)
\end{aligned}
$$

whose verifications, very easy, are omitted.

For part (b), let $\mu_{N, i}(\cdot / \sigma)$ be the conditional probability law of $\sigma(i)$ knowing $\sigma(j), j \neq i$. Then

$$
\mu_{N, i}(0 / \sigma)=\frac{e^{-\beta H^{N}\left(\sigma^{i-}\right)}}{e^{-\beta H^{N}\left(\sigma^{i-}\right)}+\left(e^{z\left|A_{i}^{N}\right|}-1\right) e^{-\beta H^{N}\left(\sigma^{i+}\right)}} .
$$


By an easy calculus and the fact that

$$
\frac{\mu_{N, i}(1 / \sigma)}{\mu_{N, i}(0 / \sigma)}=\left(e^{z\left|A_{i}^{N}\right|}-1\right) e^{-\beta\left(D_{i}^{+} H^{N}\right)\left(\sigma^{i-}\right)},
$$

we have

$$
\begin{aligned}
\mu_{N, i}\left(g\left[D_{i}^{-} f+\left(e^{z\left|A_{i}^{N}\right|}-1\right) e^{-\beta D_{i}^{+} H^{N}} D_{i}^{+} f\right] / \sigma\right) & =\mu_{N, i}\left(D_{i}^{-} f \cdot D_{i}^{-} g / \sigma\right) \\
& =\left(e^{z\left|A_{i}^{N}\right|}-1\right) \mu_{N, i}\left(e^{-\beta D_{i}^{+} H^{N}} D_{i}^{+} f \cdot D_{i}^{+} g / \sigma\right)
\end{aligned}
$$

where (4.5) follows by at first taking the conditional expectation.

Let

$$
\begin{aligned}
& \mathcal{D}^{N}:=\left\{f\left(q_{N} \omega\right) ; N \geqslant 1, f:\{0,1\}^{2^{N}} \rightarrow \mathbb{R}\right\}, \\
& \mathcal{D}_{0}:=\bigcup_{N \geqslant 1} \mathcal{D}^{N} .
\end{aligned}
$$

This space of test-functions $\mathcal{D}_{0}$ contains $\mathcal{D}_{1}$ associated with the algebra $\mathcal{A}$ generated by $\bigcup_{N \geqslant 1} \mathcal{P}^{N}$, given in Proposition 3.1 (3.2). Remark that for any $F(\omega)=f\left(q_{N} \omega\right)$ in $\mathcal{D}^{N}$, as $q_{N} \omega$ is a function of $q_{N+1}(\omega)$, we have $F \in \mathcal{D}^{N+1}$. Then for any $F \in \mathcal{D}_{0}$, for all $N$ sufficiently large, there is a unique function $f_{N}:\{0,1\}^{I_{N}} \rightarrow \mathbb{R}$ such that $F(\omega)=f_{N}\left(q_{N} \omega\right), \forall \omega \in \Omega_{\Lambda}$.

Lemma 4.2. Assume, besides (2.1) and (2.2), moreover that $\varphi: \mathbb{R}^{d} \rightarrow(-\infty,+\infty]$ is continuous and is of finite range. Then there exists a sequence of $\mathcal{F}_{\Lambda}$-functions $\varepsilon_{n}(\omega) \rightarrow 0, \forall \omega \in \Omega_{\Lambda}$ and bounded by some constant $L$ (depending only on the partitions and $|\Lambda|$ ), such that for any $F(\omega)=f_{n}\left(q_{n} \omega\right) \in \mathcal{D}_{0}$,

$$
\left|\left(\mathcal{L}_{n} f_{n}\right)\left(q_{n} \omega\right)-\mathcal{L}_{\Lambda}^{\eta} F(\omega)\right| \leqslant \varepsilon_{n}(\omega)\|F\|_{u}\left(1+N_{\Lambda}(\omega)\right) e^{2 B \beta N_{\Lambda}(\omega)-\beta \inf _{x \in \Lambda} h^{\eta}(x)}, \quad \forall \omega \in \Omega_{\Lambda}
$$

and the factor $\exp \left(2 B \beta N_{\Lambda}(\omega)-\beta \inf _{x \in \Lambda} h^{\eta}(x)\right)$ above can be replaced by $e^{2 B \beta}$ under (C1) or (C2) in Theorem 2.1.

Proof. At first notice that

$$
\left|\sum_{i=1}^{2^{n}} 1_{\omega\left(A_{i}^{n}\right)>1}\left(D_{i}^{-} f_{n}\right)\left(q_{n} \omega\right)\right| \leqslant 2\|F\|_{u} N_{\Lambda}(\omega)\left(1-1_{\Omega_{\Lambda}^{n}}(\omega)\right) .
$$

On the other hand, we have by (2.9), $D_{x}^{+} H_{\Lambda}^{\eta}\left(p_{n} \omega\right) \geqslant-2 B N_{\Lambda}(\omega)+\inf _{x \in \Lambda} h^{\eta}(x)$. Then

$$
\begin{aligned}
& \sum_{i \in I_{N}}\left|\frac{e^{z\left|A_{i}^{N}\right|}-1}{\left|A_{i}^{N}\right|} \int_{A_{i}^{N}} e^{-\beta D_{x}^{+} H_{\Lambda}^{\eta}\left(p_{n} \omega\right)} D_{x}^{+} F(\omega) d x-z \int_{A_{i}^{N}} e^{-\beta D_{x}^{+} H_{\Lambda}^{\eta}(\omega)} D_{x}^{+} F(\omega) d x\right| \\
& \leqslant \max _{i \in I_{N}}\left|\frac{e^{z\left|A_{i}^{N}\right|}-1}{\left|A_{i}^{N}\right|}-z\right| 2\|F\|_{u}|\Lambda| e^{2 B \beta N_{\Lambda}(\omega)-\beta \inf _{x \in \Lambda} h^{\eta}(x)} \\
& \quad+2\|F\|_{u} \int_{\Lambda}\left|e^{-\beta D_{x}^{+} H_{\Lambda}^{\eta}\left(p_{n} \omega\right)}-e^{-\beta D_{x}^{+} H_{\Lambda}^{\eta}(\omega)}\right| z d x,
\end{aligned}
$$

where the last factor is bounded by $2 \exp \left(2 B \beta N_{\Lambda}(\omega)-\beta \inf _{x \in \Lambda} h^{\eta}(x)\right)$ and tends to zero, because $p_{n} \omega \rightarrow \omega$ weakly for all $\omega$, and $H_{\Lambda}^{\eta}(\omega)$ is continuous on $\omega$ for the weak convergence topology in $\Omega_{\Lambda}$ by our assumption 
on $\varphi$. Those estimates yield the desired estimate by the expressions of $\mathcal{L}_{\Lambda}^{\eta} F$ and Lemma 4.1(a). Finally in the context of (C1) or (C2) in Theorem 2.1, it is enough to use $D_{x}^{+} H_{\Lambda}^{\eta}\left(p_{n} \omega\right) \geqslant-2 B$ in the estimates above.

\subsection{A priori estimate of Ligget for the discrete spin model}

Assume now (C1) or (C2) in Theorem 2.1. The generator $\mathcal{L}_{N}$ in (4.1) can be written as

$$
\mathcal{L}_{N} f(\sigma)=\sum_{i \in I_{N}} \int_{\{0,1\}} c_{i}(\sigma, d \xi)\left(f\left(\sigma^{i, \xi}\right)-f(\sigma)\right)
$$

where $\sigma^{i, \xi}(j)=\sigma(j)$ for $j \neq i$ and $\sigma^{i, \xi}(i)=\xi$, and

$$
c_{i}(\sigma, 0)=1_{\sigma(i)=1}, \quad c_{i}(\sigma, 1)=1_{\sigma(i)=0}\left(e^{z\left|A_{i}^{N}\right|}-1\right) e^{-\beta D_{i}^{+} H^{N}(\sigma)} .
$$

According to Ligget [11], p. 24, introduce the matrix $\Gamma_{N}:=\left(\Gamma_{N}(i, j)\right)_{i, j \in I_{N}}$ of Ligget, where $\Gamma_{N}(i, i):=0$ and for all $i \neq j\left(\in I_{N}\right)$

$$
\begin{aligned}
\Gamma_{N}(i, j):= & \sup _{\sigma=\tau \text { off } j}\left\|c_{i}(\sigma, \cdot)-c_{i}(\sigma, \cdot)\right\| \\
= & \sup _{\sigma=\tau \text { off } j, \sigma(i)=\tau(i)=0}\left(e^{z\left|A_{i}^{N}\right|}-1\right)\left|e^{-\beta D_{i}^{+} H^{N}(\sigma)}-e^{-\beta D_{i}^{+} H^{N}(\tau)}\right| \\
= & \left(e^{z\left|A_{i}^{N}\right|}-1\right)\left|1-e^{-\beta \varphi\left(x_{j}^{N}-x_{i}^{N}\right)}\right| \\
& \quad \times \sup _{\sigma} \exp \left(-\beta\left(\sum_{k \neq i, j} \sigma(k) \varphi\left(x_{k}^{N}-x_{i}^{N}\right)+\sum_{y \in \operatorname{supp} \eta_{\Lambda^{c}}} \varphi\left(y-x_{i}^{N}\right)\right)\right) .
\end{aligned}
$$

Under (C1) or (C2) in Theorem 2.1, the last factor above is bounded by $e^{2 B \beta}$ (without (C1) or (C2), the last factor explodes!), then

$$
\Gamma_{N}(i, j) \leqslant\left(e^{z\left|A_{i}^{N}\right|}-1\right)\left|1-e^{-\beta \varphi\left(x_{j}^{N}-x_{i}^{N}\right)}\right| \cdot e^{2 B \beta} .
$$

Let $\nabla_{f}(i)=\sup _{\sigma}\left|f\left(\sigma^{i+}\right)-f\left(\sigma^{i-}\right)\right|, \nabla_{f}:=\left(\nabla_{f}(i)\right)_{i \in I_{N}}$ (column vector), and

$$
\left\|\nabla_{f}\right\|_{\infty}:=\max _{i \in I_{N}} \nabla_{f}(i), \quad\left\|\nabla_{f}\right\|_{1}:=\sum_{i \in I_{N}} \nabla_{f}(i) .
$$

We now translate Ligget's $M-\varepsilon$ theorem ([11], Chapter I, Theorem 3.9) into the

Theorem 4.3. Let $P_{t}^{N}:=e^{t \mathcal{L}_{N}}$. Assume $(\mathrm{C} 1)$ or $(\mathrm{C} 2)$ in Theorem 2.1 and $\varphi$ is continuous and of finite range. Then

(a) (Ligget) $\nabla_{P_{t}^{N} f}(j) \leqslant e^{-t}\left[e^{t\left(\Gamma_{N}\right)^{*}} \nabla_{f}\right](j), \forall j \in I_{N}, t \geqslant 0$, where $\left(\Gamma_{N}\right)^{*}$ denotes the transposition of the matrix $\Gamma_{N}$.

(b) Let $M:=M(z, \beta, B):=z e^{2 B \beta} \int_{\mathbb{R}^{d}}\left|1-e^{-\beta \varphi(x)}\right| d x$. For any $\delta>0$, for all $N$ sufficiently large, we have for all $f:\{0,1\}^{I_{N}} \rightarrow \mathbb{R}$,

$$
\left\|\nabla_{P_{t}^{N} f}\right\|_{\infty} \leqslant e^{-(1-M-\delta) t}\left\|\nabla_{f}\right\|_{\infty}, \quad \forall t \geqslant 0 .
$$

(c) Assume moreover that $\max _{i \in I_{N}}\left|A_{i}^{N}\right| \leqslant(1+\varepsilon(N)) \min _{i \in I_{N}}\left|A_{i}^{N}\right|$ where $\varepsilon(N) \rightarrow 0$ as $N \rightarrow \infty$ (this is possible for e.g. $\Lambda$ rectangles). Then for any $\delta>0$, for all $N$ sufficiently large, we have for all $f:\{0,1\}^{I_{N}} \rightarrow \mathbb{R}$,

$$
\left\|\nabla_{P_{t}^{N}}\right\|_{1} \leqslant e^{-(1-M-\delta) t}\left\|\nabla_{f}\right\|_{1}, \quad \forall t \geqslant 0 .
$$


Recall that $\left\|\nabla_{f}\right\|_{1}$ is the "triple" norm of Ligget.

Proof. Part (a) is exactly [11], Chapter I, Theorem 3.9(c) since the constant $\varepsilon$ given there is $\geqslant 1$ for the model here.

For part (b), notice that for any $h \in l^{\infty}\left(I_{N}\right)$ identified as column vector,

$$
\left|\left(\Gamma_{N}\right)^{*} h(j)\right|=\left|\sum_{i \in I_{N}} \Gamma_{N}(i, j) h(i)\right| \leqslant\|h\|_{\infty} \cdot \sum_{i \in I_{N}}\left(e^{z\left|A_{i}^{N}\right|}-1\right)\left|1-e^{-\beta \varphi\left(x_{j}^{N}-x_{i}^{N}\right)}\right| \cdot e^{2 B \beta}
$$

by (4.9). Thus

$$
\begin{aligned}
\limsup _{N \rightarrow \infty}\left\|\left(\Gamma_{N}\right)^{*}\right\|_{l^{\infty}\left(I_{N}\right)} & \leqslant \limsup _{N \rightarrow \infty} \max _{j \in I_{N}} \sum_{i \in I_{N}}\left(e^{z\left|A_{i}^{N}\right|}-1\right)\left|1-e^{-\beta \varphi\left(x_{j}^{N}-x_{i}^{N}\right)}\right| \cdot e^{2 B \beta} \\
& \leqslant z e^{2 B \beta} \int_{\mathbb{R}^{d}}\left|1-e^{-\beta \varphi(x)}\right| d x=M
\end{aligned}
$$

where part (b) follows from (a) by noting that $\left\|\exp \left(t\left(\Gamma_{N}\right)^{*}\right)\right\|_{l^{\infty}\left(I_{N}\right)} \leqslant \exp \left(t\left\|\Gamma_{N}^{*}\right\|_{l^{\infty}\left(I_{N}\right)}\right)$.

Finally for part (c), we have by a similar calculus

$$
\begin{aligned}
\left\|\left(\Gamma_{N}\right)^{*}\right\|_{l^{1}\left(I_{N}\right)} & \leqslant \max _{i \in I_{N}} \sum_{j \in I_{N}}\left(e^{z\left|A_{i}^{N}\right|}-1\right)\left|1-e^{-\beta \varphi\left(x_{j}^{N}-x_{i}^{N}\right)}\right| \cdot e^{2 B \beta} \\
& \leqslant(1+\varepsilon(N)) \max _{i \in I_{N}} \sum_{j \in I_{N}}\left(e^{z\left|A_{j}^{N}\right|}-1\right)\left|1-e^{-\beta \varphi\left(x_{j}^{N}-x_{i}^{N}\right)}\right| \cdot e^{2 B \beta} .
\end{aligned}
$$

Then

$$
\limsup _{N \rightarrow \infty}\left\|\left(\Gamma_{N}\right)^{*}\right\|_{l^{1}\left(I_{N}\right)} \leqslant z e^{2 B \beta} \int_{\mathbb{R}^{d}}\left|1-e^{-\beta \varphi(x)}\right| d x=M
$$

where part (c) follows.

\section{Exponential decay of the Glauber dynamics and proof of the main result}

\subsection{Exponential decay of the Glauber dynamics}

The following theorem, being a consequence of Ligget's Theorem 4.3 and of Proposition 3.2, is our second main result. It gives an exponential convergence of $P_{t}^{\Lambda, \eta}(\sigma, \cdot)$ to $\mu_{\Lambda}^{\eta}$ w.r.t. the norms different from that of $L^{2}\left(\mu_{\Lambda}^{\eta}\right)$, for every starting point, and it is in reality stronger than the spectral gap result in Theorem 2.1.

Theorem 5.1. Assume $(\mathrm{C} 1)$ or $(\mathrm{C} 2)$ in Theorem 2.1. Let $\left(P_{t}^{\Lambda, \eta}=P_{t}\right)$ be the transition semigroup on $b \mathcal{F}_{\Lambda}$ generated by $\mathcal{L}_{\Lambda}^{\eta}$ (constructed in Proposition 3.2).

(a) For any $F=f\left(\omega\left(B_{1}\right), \ldots, \omega\left(B_{k}\right)\right)$ where $k \geqslant 1, B_{k} \in \mathcal{B}(\Lambda), f: \mathbb{N}^{k} \rightarrow \mathbb{R}$ is bounded,

$$
\left\|D_{.}^{+} P_{t}^{\Lambda, \eta} F\right\|_{u} \leqslant e^{-t(1-M)}\left\|D_{.}^{+} F\right\|_{u}, \quad \forall t \geqslant 0
$$

where $M:=z e^{2 B \beta} \int_{\mathbb{R}^{d}}\left|1-e^{-\beta \varphi(x)}\right| d x$, and $\left\|D^{+} F\right\|_{u}:=\sup _{(x, \omega) \in \Lambda \times \Omega_{\Lambda}}\left|D_{x}^{+} F(\omega)\right|$. 
(b) If $\Lambda$ allows a sequence of partitions $\left(\mathcal{P}^{N}\right)_{N \geqslant 1}$ as in Section 4 such that $\max _{i}\left|A_{i}^{N}\right| \leqslant(1+\varepsilon(N)) \min _{i}\left|A_{i}^{N}\right|$ where $\varepsilon(N) \rightarrow 0$ (e.g. $\Lambda$ rectangles), then for all $F=f\left(\omega\left(B_{1}\right), \ldots, \omega\left(B_{k}\right)\right)$ where $k \geqslant 1, B_{k} \in \mathcal{A}$ (the algebra generated by $\left.\left\{\mathcal{P}^{N}, N \geqslant 1\right\}\right), f: \mathbb{N}^{k} \rightarrow \mathbb{R}$ is bounded,

$$
\int_{\Lambda} d x\left\|D_{x}^{+} P_{t}^{\Lambda, \eta} F\right\|_{u} \leqslant e^{-t(1-M)} \int_{\Lambda} d x\left\|D_{x}^{+} F\right\|_{u}, \quad \forall t \geqslant 0 .
$$

Remarks 5.2. It would be interesting to investigate whether the Ligget $M-\varepsilon$ theorem still holds true for general continuous gas models, with a direct proof. The classical proof of Ligget [11] in the lattice case (i.e., on $E^{T}$ with $T$ at most countable) relies on the Yosida theorem (for applying it, Ligget assumed the compactness of spin $E$ and the continuity of local transition rate kernels for proving the uniqueness in $C_{b}\left(E^{T}\right)$ ). For the continuous gas models, the main difficulty for a direct proof seems residing in the facts that

(i) Even in the finite volume case, the configurations space $\Omega_{\Lambda}$ is unbounded and infinite dimensional;

(ii) the Yosida theorem is no longer available, because usual Markov semigroups $\left(P_{t}\right)$ on $\Omega_{\Lambda}$ is not strongly continuous on $C_{b}\left(\Omega_{\Lambda}\right)$ (e.g., the free semigroup $\left(P_{t}^{0}\right)$ is such an example). The uniqueness in Proposition 3.2 and its extension to more general models might be helpful in this story.

Remarks 5.3. In the lattice case, restricted to a finite number of sites, Ligget [11] succeeded to obtain the exponential convergence in the uniform norm from his $M-\varepsilon$ theorem. That is impossible for the continuous gas model here, because even the free semigroup $\left(P_{t}^{0}\right)$ (i.e., $\varphi=0$ ) on a bounded domain $\Lambda$ does not have the exponential convergence in the uniform norm. In fact let $\left(\left(X_{t}^{0}\right), \mathbb{P}_{\omega}^{0}\right)$ be the free Glauber Markov process associated with $\left(P_{t}^{0}\right)$. Then $\left(N_{\Lambda}\left(X_{t}\right)\right)_{t} \geqslant 0$ is a birth-death process valued in $\mathbb{N}$ with death rate $b_{n}=n$ and birth rate $a_{n}=z|\Lambda|$, it is non-Doeblin recurrent by Mao [14], then no exponential convergence in the uniform norm.

The following elementary lemma will be used.

Lemma 5.4. Let $f: \mathbb{R}^{d} \rightarrow(-\infty,+\infty]$ be a measurable function such that $f^{-} \in L_{\mathrm{loc}}^{1}\left(\mathbb{R}^{d}, d x\right)$. Then

$$
\lim _{\varepsilon \rightarrow 0} h_{\varepsilon} * f(x)=f(x), \quad d x \text {-a.e. in }(-\infty,+\infty]
$$

where $h_{\varepsilon}(x):=\frac{1}{\varepsilon^{d}} h\left(\frac{y-x}{\varepsilon}\right), 0 \leqslant h \in C^{\infty}\left(\mathbb{R}^{d}\right)$ is an even function such that $h(x)=0$ for $|x|>1$ and $\int h(x) d x=1$.

Proof. This lemma is well known if $f \in L_{\mathrm{loc}}^{1}\left(\mathbb{R}^{d}, d x\right)$ (Lebesgue's theorem). We can then assume that $f=f^{+} \geqslant$ 0 . For any $L>0$, we have for $d x$-a.e. $x$,

$$
\liminf _{\varepsilon \rightarrow 0} h_{\varepsilon} * f(x) \geqslant \liminf _{\varepsilon \rightarrow 0} h_{\varepsilon} *(f \wedge L)(x)=f(x) \wedge L
$$

and on the other hand by Jensen's inequality, we have for $d x$-a.e. $x$,

$$
\liminf _{\varepsilon \rightarrow 0} e^{-h_{\varepsilon} * f}(x) \leqslant \liminf _{\varepsilon \rightarrow 0}\left(h_{\varepsilon} * e^{-f}\right)(x)=e^{-f(x)} .
$$

Proof of Theorem 5.1. (a). We separate its proof into three steps of approximation.

Step 1. The first approximation. We assume at first that $\varphi$ is moreover continuous and of finite range and $F \in \mathcal{D}_{0}$. Write $P_{t}:=P_{t}^{\Lambda, \eta}$ and $P_{t}^{N}:=e^{t \mathcal{L}_{N}}$ for simplicity. For any $F \in \mathcal{D}_{0}, F(\omega)=f_{n}\left(q_{n} \omega\right)$ for all $n \geqslant N$ (for some $N$ ), where $f_{n}:\{0,1\}^{I_{n}} \rightarrow \mathbb{R}$. By Proposition 3.2 (3.7), we have that for any $\omega \in \Omega_{\Lambda}$ and any $t>0$, $s \rightarrow P_{s}\left[\left(P_{t-s}^{n} f_{n}\right)\left(q_{n} \cdot\right)\right](\omega)$ is continuous differentiable on $[0, t]$, and

$$
\frac{d}{d s} P_{s}\left[\left(P_{t-s}^{n} f_{n}\right)\left(q_{n} \cdot\right)\right](\omega)=P_{s}\left(\mathcal{L}_{\Lambda}^{\eta}\left[\left(P_{t-s}^{n} f_{n}\right)\left(q_{n} \cdot\right)\right](\omega)-\left(\mathcal{L}_{n} P_{t-s}^{n} f_{n}\right)\left(q_{n} \omega\right)\right) .
$$


Thus

$$
P_{t} F(\omega)-\left(P_{t}^{n} f_{n}\right)\left(q_{n} \omega\right)=\int_{0}^{t} d s P_{s}\left[\mathcal{L}_{\Lambda}^{\eta}\left[\left(P_{t-s}^{n} f_{n}\right)\left(q_{n} \cdot\right)\right](\omega)-\left(\mathcal{L}_{n} P_{t-s}^{n} f_{n}\right)\left(q_{n} \omega\right)\right] d s .
$$

By Lemma 4.2, $\left|\mathcal{L}_{\Lambda}^{\eta}\left[\left(P_{t-s}^{n} f_{n}\right)\left(q_{n} \cdot\right)\right](\omega)-\left(\mathcal{L}_{n} P_{t-s}^{n} f_{n}\right)\left(q_{n} \omega\right)\right| \leqslant \varepsilon_{n}(\omega)\|F\|_{u}\left(1+N_{\Lambda}(\omega)\right) e^{2 B \beta}$ where $\varepsilon_{n}(\omega) \rightarrow 0$ everywhere and uniformly bounded by some constant $L$. Then by (3.9) in the proof of Proposition 3.2, we can apply the dominated convergence and get

$$
\lim _{n \rightarrow \infty}\left(P_{t}^{n} f_{n}\right)\left(q_{n} \omega\right)=P_{t} F(\omega), \quad \forall \omega .
$$

Consequently $\forall(x, \omega) \in \Lambda \times \Omega_{\Lambda}$,

$$
\begin{aligned}
\left|D_{x}^{+} P_{t} F(\omega)\right| & =\limsup _{n \rightarrow \infty}\left|D_{x}^{+}\left(P_{t}^{n} f_{n}\right)\left(q_{n} \omega\right)\right| \\
& \leqslant \limsup _{n \rightarrow \infty}\left\|\max _{i \in I_{n}}\left(D_{i}^{+} P_{t}^{n} f_{n}\right) \circ q_{n}\right\|_{u} \\
& \leqslant e^{-(1-M) t} \limsup _{n \rightarrow \infty}\left\|\max _{i \in I_{n}}\left(D_{i}^{+} f_{n}\right)\right\|_{u} \\
& =e^{-(1-M) t}\left\|D^{+} F\right\|_{u}
\end{aligned}
$$

the desired estimate, where the crucial third inequality follows by Theorem 4.3(b).

Step 2. The second approximation. Assume at first that $\varphi$ is of finite range. For any $\varepsilon \in(0,1)$ let

$$
\varphi_{\varepsilon}(x):=h_{\varepsilon} * \varphi(x):=\int_{\mathbb{R}^{d}} h_{\varepsilon}(y-x) \varphi(y) d y
$$

where $h_{\varepsilon}$ is given in Lemma 5.4. Obviously $\varphi_{\varepsilon}$ satisfies $(\mathrm{C} 1)$ or (C2) if so does $\varphi$, and $\varphi_{\varepsilon}: \mathbb{R}^{d} \rightarrow(-\infty,+\infty]$ is continuous and of finite range. By Lemma $5.4, \varphi_{\varepsilon}(x) \rightarrow \varphi(x), d x$-a.e.

Write $H_{\varepsilon}, \mu_{\varepsilon}, \mathcal{L}_{\varepsilon}, P_{t}^{\varepsilon}$ respectively for the Hamiltonian (with the same boundary condition $\eta \in \Omega^{\varphi}$ ), Gibbs measure, the generator of the Glauber dynamic and the corresponding semigroup on $b \mathcal{F}_{\Lambda}$, associated with $\varphi_{\varepsilon}$ instead of $\varphi$.

Again by Proposition 3.2, we have as step 1 that for any $F \in \mathcal{D}_{0}$,

$$
\begin{aligned}
\left|P_{t} F(\omega)-P_{t}^{\varepsilon} F(\omega)\right| & =\left|\int_{0}^{t} P_{s}\left(\mathcal{L}_{\Lambda}^{\eta}-\mathcal{L}_{\varepsilon}\right) P_{t-s}^{\varepsilon} F(\omega) d s\right| \\
& =\left|\int_{0}^{t} d s P_{s}\left(\int_{\Lambda}\left(e^{-\beta D_{x}^{+} H_{\Lambda}^{\eta}}-e^{-\beta D_{x}^{+} H_{\varepsilon}}\right) D_{x}^{+} P_{t-s}^{\varepsilon} F z d x\right)(\omega)\right| \\
& \leqslant 2\|F\|_{u} \int_{0}^{t} d s P_{s}\left(\int_{\Lambda}\left|e^{-\beta D_{x}^{+} H_{\Lambda}^{\eta}}-e^{-\beta D_{x}^{+} H_{\varepsilon}}\right| z d x\right)(\omega)
\end{aligned}
$$

where it follows by dominated convergence that $P_{t}^{\varepsilon} F(\omega) \rightarrow P_{t} F(\omega)$ as $\varepsilon \rightarrow 0$. Now the desired result for $P_{t} F$ follows from that of $P_{t}^{\varepsilon} F$ obtained in step 1 .

We now remove the finite range condition. Putting $\varphi_{r}(x):=\varphi(x) 1_{|x|<r}$, we see that as $r \rightarrow \infty, \int_{\Lambda^{c}} \varphi_{r}(y-$ $x) \eta(d y) \rightarrow \int_{\Lambda^{c}} \varphi(y-x) \eta(d y)$ for every $x \in \mathbb{R}^{d}$ by our assumption that $\eta \in \Omega^{\varphi}$. Hence the same proof as above yields (5.1) for all $F \in \mathcal{D}_{0}$, without the finite range condition. 
Step 3. Passage to $F=f\left(\omega\left(B_{1}\right), \ldots, \omega\left(B_{k}\right)\right)$. We can choose the sequence of partitions $\left(\mathcal{P}^{N}\right)_{N \geqslant 1}$ in Section 4 so that moreover each $B_{i}$ is an union of elements in $\mathcal{P}^{N}$ for some $N$. Then for all $n \geqslant N, B_{i}=\bigcup_{j \in J_{i}^{n}} A_{j}^{n}$ where $J_{i}^{n} \subset I_{n}=\left\{1, \ldots, 2^{n}\right\}$. Let

$$
F_{n}(\omega)=f\left(\sum_{j \in J_{1}^{n}} \omega\left(A_{j}^{n}\right) \wedge 1, \ldots, \sum_{j \in J_{k}^{n}} \omega\left(A_{j}^{n}\right) \wedge 1\right) .
$$

It is easy to see that $\left|D_{x} F_{n}(\omega)\right| \leqslant\left|D_{x} F(\omega)\right|$ and $F_{n} \rightarrow F$, everywhere. Now for every $(x, \omega) \in \Lambda \times \Omega_{\Lambda}$, we have by step $2\left(\right.$ as $\left.F_{n} \in \mathcal{D}_{0}\right)$,

$$
\left|D_{x}^{+} P_{t} F(\omega)\right|=\limsup _{n \rightarrow \infty}\left|D_{x}^{+}\left(P_{t} F_{n}\right)(\omega)\right| \leqslant e^{-(1-M) t} \limsup _{n \rightarrow \infty}\left\|D_{.}^{+} F_{n}\right\|_{u} \leqslant e^{-(1-M) t}\left\|D^{+} F\right\|_{u}
$$

the desired result.

(b) It can be proved exactly in the same way as in the proof of part (a), by applying Theorem 4.3(c) instead of Theorem 4.3(b).

\subsection{Proof of Theorem 2.1}

Proof of the upper bound in (2.12). By Proposition 3.1, $N_{\Lambda} \in \mathbb{D}\left(\mathcal{E}_{\Lambda}^{\eta}\right)$ and

$$
\mathcal{E}_{\Lambda}^{\eta}\left(N_{\Lambda}, N_{\Lambda}\right)=\int d \mu_{\Lambda}^{\eta}(\omega) \int_{\Lambda}\left(D_{x}^{-} N_{\Lambda}(\omega)\right)^{2} \omega(d x)=\mu_{\Lambda}^{\eta}\left(N_{\Lambda}\right) .
$$

Then

$$
\lambda_{1} \leqslant \frac{\mu_{\Lambda}^{\eta}\left(N_{\Lambda}\right)}{\mu_{\Lambda}^{\eta}\left(N_{\Lambda}, N_{\Lambda}\right)} \leqslant 1+z e^{\beta B} \int_{\mathbb{R}^{d}}\left(1-e^{-\beta \varphi^{+}(x)}\right) d x
$$

where the last inequality is a classical estimate, see [17], Proposition 3.4.9.

Proof of the lower bound in (2.12). Obviously we may assume without loss of generality that $M:=$ $z e^{2 B \beta} \int_{\mathbb{R}^{d}}\left|1-e^{\varphi(x)}\right| d x<1$.

Let $-\mathcal{L}_{\Lambda}^{\eta}=\int_{\mathbb{R}^{+}} \lambda d E_{\lambda}$ be the spectral decomposition of $-\mathcal{L}_{\Lambda}^{\eta}$ in $L^{2}\left(\mu_{\Lambda}^{\eta}\right)$ (recall that $E_{0} F=\mu_{\Lambda}^{\eta}(F)$ ). Let $\lambda_{0}$ be an arbitrary point in $(0,1-M)$. By Theorem 5.1, for any $F \in \mathcal{D}_{0}$ given in (4.8),

$$
\begin{aligned}
e^{-2 \lambda_{0} t} \int_{\left[0, \lambda_{0}\right]} \lambda d\left\langle\left(E_{\lambda}-E_{0}\right) F, F\right\rangle & \leqslant \int_{(0,+\infty)} \lambda e^{-2 \lambda t} d\left\langle E_{\lambda} F, F\right\rangle=\mathcal{E}_{\Lambda}^{\eta}\left(P_{t} F, P_{t} F\right) \\
& =\mu_{\Lambda}^{\eta}\left(\int_{\Lambda} e^{-\beta D_{x}^{+} H_{\Lambda}^{\eta}(\omega)}\left(D_{x}^{+} P_{t} F\right)^{2} z d x\right) \\
& \leqslant z|\Lambda| e^{2 B \beta}\left\|D_{.}^{+} P_{t} F\right\|_{u}^{2} \\
& \leqslant z|\Lambda| e^{2 B \beta}\left\|D_{.}^{+} F\right\|_{u}^{2} \cdot e^{-2(1-M) t}
\end{aligned}
$$

where it follows that $\int_{\left[0, \lambda_{0}\right]} \lambda d\left(E_{\lambda}-E_{0}\right) F=0$, by letting $t \rightarrow \infty$ (because $\left\|D_{.}^{+} F\right\|_{u}=0$ implies $F=\mu_{\Lambda}^{\eta}(F)$ ). As $\mathcal{D}_{0}$ is dense in $L^{2}\left(\mu_{\Lambda}^{\eta}\right)$ and $\int_{\left[0, \lambda_{0}\right]} \lambda d\left(E_{\lambda}-E_{0}\right)$ is bounded on $L^{2}\left(\mu_{\Lambda}^{\eta}\right)$, then $\int_{\left[0, \lambda_{0}\right]} \lambda d\left(E_{\lambda}-E_{0}\right)=0$, i.e., $E_{\lambda}=E_{0}$ for all $\lambda \in\left[0, \lambda_{0}\right]$. As $\lambda_{0} \in(0,1-M)$ is arbitrary, then

$$
\lambda_{1}=\inf \left\{\lambda>0 ; E_{\lambda}-E_{0} \neq 0\right\} \geqslant 1-M
$$

the desired result. 


\subsection{Extension to other Glauber dynamics}

One can transfer the estimate of $\lambda_{1}$ in Theorem 2.1 to other reversible or non-reversible Glauber dynamics associated with $\mu_{\Lambda}^{\eta}$, by comparison (always under (C1) or (C2)). At first if $\left(P_{t}\right)$ is a Markov semigroup on $L^{2}\left(\mu_{\Lambda}^{\eta}\right)$ with invariant measure $\mu_{\Lambda}^{\eta}$ and with generator $\mathcal{L}$ (representing a Glauber dynamic), let $\lambda_{1}(P$.) be the largest exponential convergence rate of $P_{t}$ to the equilibrium $\mu_{\Lambda}^{\eta}$, i.e., the best constant such that

$$
\mu_{\Lambda}^{\eta}\left(P_{t} F, P_{t} F\right) \leqslant e^{-2 \lambda_{1} t} \mu_{\Lambda}^{\eta}(F, F), \quad \forall F \in L^{2}\left(\mu_{\Lambda}^{\eta}\right) .
$$

As this last property is equivalent to the Poincaré inequality

$$
\lambda_{1} \mu_{\Lambda}^{\eta}(f, f) \leqslant\langle F,-\mathcal{L} F\rangle_{\mu_{\Lambda}^{\eta}}, \quad \forall F \in \operatorname{Dom}(\mathcal{L})
$$

hence $\lambda_{1}$ coincides with the spectral gap of $\left(\mathcal{L}+\mathcal{L}^{*}\right) / 2$ (in the form sense). In other words we are always reduced to the reversible case.

For constructing reversible Glauber dynamics, consider the pre-Dirichlet form

$$
\mathcal{E}_{Z}(F, G):=\int_{\Omega_{\Lambda}} d \mu_{\Lambda}^{\eta}(\omega) \int_{\Lambda} e^{Z(x, \omega)-\beta D_{x}^{+} H_{\Lambda}^{\eta}(\omega)} D_{x}^{+} F(\omega) D_{x}^{+} G(\omega) z d x, \quad \forall F, G \in b \mathcal{F}_{\Lambda}
$$

where $Z(x, \omega)$ is $\mathcal{B}(\Lambda) \otimes \mathcal{F}_{\Lambda}$-measurable, such that

(i) $Z(x, \omega) \geqslant C(Z)$;

(ii) $Z(x, \omega)-\beta D_{x}^{+} H_{\Lambda}^{\eta}(\omega)$ is upper bounded.

Then from Theorem 2.1, we have

$$
(1-M) e^{-C(Z)} \mu_{\Lambda}^{\eta}(F, F) \leqslant \mathcal{E}_{Z}(F, F), \quad \forall F \in b \mathcal{F}_{\Lambda} .
$$

If we assume moreover that

(iii) $\int_{\Lambda} e^{Z(x, \omega)} d \omega(x) \in L^{2}\left(\mu_{\Lambda}^{\eta}\right)$,

then by the duality formula (3.1), the associated symmetric (nonpositive definite) pre-generator is given by

$$
\mathcal{L}_{Z} F(\omega)=\int_{\Lambda} e^{Z\left(x, \epsilon_{x}^{-} \omega\right)} D_{x}^{-} F d \omega(x)+\int_{\Lambda} e^{Z(x, \omega)-\beta D_{x}^{+} H_{\Lambda}^{\eta}(\omega)} D_{x}^{+} F(\omega) z d x, \quad \forall F \in b \mathcal{F}_{\Lambda} .
$$

Thus $\left(\mathcal{E}_{Z}, b \mathcal{F}_{\Lambda}\right)$ is closable and its closure will be still denoted by $\mathcal{E}_{Z}$. Its spectral gap $\lambda_{1}\left(\mathcal{E}_{Z}\right)$ verifies then

$$
\lambda_{1}\left(\mathcal{E}_{Z}\right) \geqslant(1-M) e^{-C(Z)} .
$$

As in Section 2, one can prove that $\left(\mathcal{L}_{Z}, b \mathcal{F}_{\Lambda}\right)$ is essentially self-adjoint in $L^{2}\left(\mu_{\Lambda}^{\eta}\right)$ and its closure generates a conservative reversible Glauber dynamic.

A quite natural choice of $Z$ is $Z_{\delta}(x, \omega)=\beta \delta D_{x}^{+} H(\omega)$ with $\delta \in[0,1]$ for which all conditions (i), (ii) and (iii) are satisfied under $(\mathrm{C} 1)$ or $(\mathrm{C} 2)$. For this last family of $Z_{\delta}$, one can prove that all results in Section 3 remain true; but surprisely enough, if $\delta>0$, in the process of approximation of $\mathcal{L}_{Z_{\delta}}$ by discrete models as in Section 4, though the constant $\varepsilon$ in the Ligget's $M-\varepsilon$ theorem might become very large, but $M$ explodes as well as $M-\varepsilon$ in general. That's why we choose $\delta=0$ in this paper.

Note added to the revised version: I learn from A. Guillin during Augest ( 6 monthes after the submission of this paper), the work by Kondratiev and Lytvynov [13] (June 2003) who prove independently the same lower bound of the spectral gap when $\varphi \geqslant 0$. 


\section{Acknowledgements}

I am grateful to the referees for their conscientious comments and correction of type mistakes.

\section{References}

[1] C. Ané, M. Ledoux, On logarithmic Sobolev inequalities for continuous random walks on graphs, Probab. Theory Related Fields 116 (2000) 573-602.

[2] T. Bodineau, B. Heffler, The log-Sobolev inequality for unbounded spin systems, J. Funct. Anal. 166 (1) (1999) $168-178$.

[3] T. Bodineau, B. Heffler, Correlation, spectral gap and log-Sobolev inequalities for unbounded spin systems, in: Differential Equations and Math. Phys., Amer. Math. Soc., Providence, RI, 2000, pp. 51-66.

[4] L. Bertini, N. Cancrini, F. Cesi, The spectral gap for a Glauber-type dynamics in a continuous gas, Ann. Inst. H. Poincaré PR 38 (1) (2002) 91-108.

[5] F. Cesi, Quasi-factorisation of entropy and log-Sobolev inequalities for Gibbs random fields, Probab. Theory Related Fields 120 (2001) $569-584$.

[6] P. Dai Pra, A.M. Paganoni, G. Posta, Entropy inequalities for unbounded spin systems, Ann. Probab. 30 (4) (2000) 1959-1976.

[7] A. Holley, D.W. Stroock, Nearest neighbor birth and death processes on the real line, Acta Mathematica 140 (1978) 103-154.

[8] O. Kallenberg, Foundations of Modern Probability, Springer-Verlag, 1997.

[9] F. Martinelli, Lectures on Glauber dynamics for discrete spin models, in: Ecole d'Eté de Saint-Flour (1997), in: Lect. Notes in Math., vol. 1717, Springer, 1999, pp. 93-191.

[10] M. Ledoux, Logarithmic Sobolev inequalities for unbounded spin systems revisited, in: Séminaire de Probabilités, in: Lect. Notes Math., vol. 1755, Springer, 2001, pp. 167-194.

[11] T.M. Ligget, Interacting Particle Systems, Springer-Verlag, 1985.

[12] S.L. Lu, H.T. Yau, Spectral gap and logarithmic Sobolev inequality for Kawasaki and Glauber dynamics, Comm. Math. Phys. 156 (1993) 399-433.

[13] Yu. Kondratiev, E. Lytvynov, Glauber dynamics of continuous particle systems, Preprint, 2003.

[14] Y.H. Mao, Strong ergodicity for Markov processes by coupling method, J. Appl. Probab. 39 (4) (2002) 839-852.

[15] S. Olla, C. Tremoulet, Equilibrium fluctuations for interacting Ornstein-Uhlenbeck particles, Comm. Math. Phys. (2003).

[16] J. Picard, Formule de dualité sur l'espace de Poisson, Ann. Inst. H. Poincaré (Prob. Stat.) 32 (4) (1996) 509-548.

[17] D. Ruelle, Statistical Mechanics: Rigorous Results, Benjamin, 1969.

[18] D.W. Stroock, B. Zegarlinski, The equivalence between the logarithmic Sobolev inequality and the Dobrushin-Shlosman mixing condition, Comm. Math. Phys. 144 (1992) 303-323.

[19] D.W. Stroock, B. Zegarlinski, The logarithmic Sobolev inequality for discrete spin systems on the lattice, Comm. Math. Phys. 149 (1992) 175-193.

[20] D. Surgailis, On the multiple Poisson stochastic integrals and associated Markov semigroups, Probab. Math. Stat. 3 (1984) $217-239$.

[21] L. Wu, A new modified logarithmic Sobolev inequality for Poisson point processes and several applications, Probab. Theory Related Fields 118 (2000) 427-438.

[22] L. Wu, Uniqueness of Nelson's diffusions, Probab. Theory Related Fields 114 (1999) 549-585.

[23] N. Yoshida, The equivalence of the logarithmic Sobolev inequality and a mixing condition for unbounded spin systems on the lattice, Ann. Inst. H. Poincaré (Prob. Stat.) 37 (2001) 223-243. 